\title{
Early Detection of Sporadic Pancreatic Cancer
}

\author{
Summative Review
}

\author{
Suresh T. Chari, MD, * Kimberly Kelly, PhD, $†$ Michael A. Hollingsworth, PhD, $\$ \$$ Sarah P. Thayer, MD, PhD, // \\ David A. Ahlquist, MD, * Dana K. Andersen, MD, $\mid$ Surinder K. Batra, PhD, + Teresa A. Brentnall, MD,\# \\ Marcia Canto, MD, MHS, ** Deborah F. Cleeter, EdD, $\uparrow$ Matthew A. Firpo, PhD, + t \\ Sanjiv Sam Gambhir, MD, PhD, \&\$ Vay Liang W. Go, MD,//// O. Joe Hines, MD, FACS, 9 / \\ Barbara J. Kenner, PhD,\#\# David S. Klimstra, MD, *** Markus M. Lerch, MD, †† Michael J. Levy, MD,//

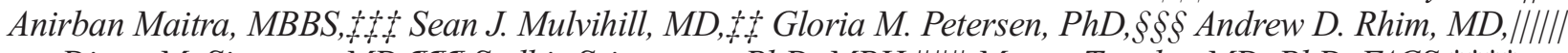 \\ Diane M. Simeone, MD, 199 Sudhir Srivastava, PhD, MPH,\#\#\# Masao Tanaka, MD, PhD, FACS, **** \\ Aaron I. Vinik, MD, PhD, FCP, MACP, FACE, ††† and David Wong, DMD, DMSct+tt
}

\begin{abstract}
Pancreatic cancer (PC) is estimated to become the second leading cause of cancer death in the United States by 2020. Early detection is the key to improving survival in PC. Addressing this urgent need, the Kenner Family Research Fund conducted the inaugural Early Detection of Sporadic Pancreatic Cancer Summit Conference in 2014 in conjunction with the 45th Anniversary Meeting of the American Pancreatic Association and Japan Pancreas Society. This seminal convening of international representatives from science, practice, and clinical research was designed to facilitate challenging interdisciplinary conversations to generate innovative ideas leading to the creation of a defined collaborative strategic pathway for the future of the field. An in-depth summary of current efforts in the field, analysis of gaps in specific areas of expertise, and challenges that exist in early detection is presented within distinct areas of inquiry: Case for Early Detection: Definitions, Detection, Survival, and Challenges; Biomarkers for Early Detection; Imaging; and Collaborative Studies. In addition, an overview of efforts in familial PC is presented in an addendum to this article. It is clear from the summit deliberations that only strategically designed collaboration among investigators, institutions, and funders will lead to significant progress in early detection of sporadic PC.
\end{abstract}

Key Words: biomarker, diabetes, screening, familial, imaging, pancreatic ductal adenocarcinoma

(Pancreas 2015;44: 693-712)
P ancreatic ductal adenocarcinoma (PDAC), referred to in this review simply as pancreatic cancer (PC), is the predominant histologic type of cancer in sporadic and familial cancer of the pancreas. Without significant advances in early detection and treatment, $\mathrm{PC}$ is estimated to become the second leading cause of cancer death in the United States by $2020 .{ }^{1}$

Pancreatic cancer develops in 3 settings: sporadic PC (SPC), which constitutes about $90 \%$ of patients; familial PC (FPC), associated with about $7 \%$ of cases; and inherited cancer syndromes, which account for $3 \%$ of patients. ${ }^{2}$

Recent developments in science and technology have not yet resulted in improved survival. Addressing this urgent need, Kenner Family Research Fund conducted the inaugural Early Detection of Sporadic Pancreatic Cancer Summit Conference, a seminal convening of international representatives from science, practice, and clinical research. Presented in conjunction with the 45th Anniversary Meeting of the American Pancreatic Association and Japan Pancreas Society (JPS) in 2014, the summit was designed to facilitate challenging interdisciplinary conversations in order to generate innovative ideas that would lead to the creation of a defined collaborative strategic pathway for the future of the field. The outcomes from this conference will be presented in a future white paper.
From the *Department of Medicine, Mayo Clinic, Rochester, MN; $†$ Department of Biomedical Engineering, University of Virginia, Charlottesville, VA; Departments of $\$$ Biochemistry and Molecular Biology, §Pathology and Microbiology, and ||Surgery, Fred \& Pamela Buffett Cancer Center, University of Nebraska, Omaha, NE; ๆDivision of Digestive Diseases and Nutrition, National Institute of Diabetes and Digestive and Kidney Disease, National Institutes of Health, Bethesda, MD; \#Division of Gastroenterology, University of Washington, Seattle, WA; **Division of Gastroenterology, Johns Hopkins University School of Medicine, Baltimore, MD; † Sawgrass Leadership Institute, Ponte Vedra Beach, FL; \$\$Department of Surgery, University of Utah, Salt Lake City, UT; §§Department of Radiology, Stanford University School of Medicine, Stanford; \|\| Department of Medicine, David Geffen School of Medicine, and $\mathbf{9}$ General Surgery, University of California Los Angeles, Los Angeles, CA; \#\#Kenner Family Research Fund; ***Department of Pathology, Memorial Sloan Kettering Cancer Center, New York, NY; HDepartment of Internal Medicine, University of Greifswald, Greifswald, Germany; $+\$$ Division of Pathology and Laboratory Medicine, University of

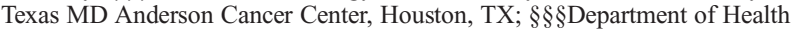
Sciences Research, Mayo Clinic, Rochester, MN; ||||||Gastroenterology Division, Department of Internal Medicine and Comprehensive Cancer Center, and 9 ब Department of Surgery, School of Medicine, University of Michigan, Ann Arbor, MI; \#\#\#Cancer Biomarkers Research Group, Division of Cancer Prevention, National Cancer Institute, Rockville, MD; ****Departments of Surgery and Oncology, Kyushu University, Fukuoka, Japan; $\dagger+\dagger$ Department of Medicine, Eastern Virginia Medical School, Norfolk, VA; and $\$+t+$ Division of Oral Biology and Medicine, CLA School of Dentistry, Jonnson Comprehensive Cancer Center, University of California Los Angeles, Los Angeles, CA. Received for publication March 20, 2015; accepted April 7, 2015.

Prepared for the Early Detection of Sporadic Pancreatic Cancer Summit Conference through the support of Kenner Family Research Fund in conjunction with the American Pancreatic Association (Stephen J. Pandol, $\mathrm{MD}$, president; Ashok Saluja, $\mathrm{PhD}$, governing board).

The authors declare no conflict of interest.

Reprints: Barbara J. Kenner, PhD, Kenner Family Research Fund, 39 E 78th St, Suite 501, New York, NY 10075 (drbkenner@kennerfamilyresearchfund.org); or Suresh T. Chari, MD, Department of Medicine, Mayo Clinic College of Medicine, 200 First St SW, Rochester, MN 55905 (chari.suresh@mayo.edu).

S.T.C., V.L.W.G., and D.S.K. are Kenner Family Research Fund scientific advisors.

S.T.C., K.K., M.A.H., and S.P.T. are section leads. Other authors are listed in alphabetical order.

Supplemental digital contents are available for this article. Direct URL citations appear in the printed text and are provided in the HTML and PDF versions of this article on the journal's Web site (www.pancreasjournal.com).

Copyright (C) 2015 Wolters Kluwer Health, Inc. All rights reserved. This is an open-access article distributed under the terms of the Creative Commons Attribution-Non Commercial-No Derivatives License 4.0 (CCBY-NC-ND), where it is permissible to download and share the work provided it is properly cited. The work cannot be changed in any way or used commercially. 
This review provides a robust collection of information articulating the current efforts in the field, the analysis of gaps in specific areas of expertise, and the challenges that exist in early detection. Four distinct panels of experts prepared presummit analyses in a foundational paper on Case for Early Detection: Definitions, Detection, Survival, and Challenges; Biomarkers for Early Detection; Imaging; and Collaborative Studies. Familial PC emerged as a theme to be presented separately and is entitled Screening in Familial Pancreatic Cancer, which is presented in an addendum to this article (Supplemental Digital Content 1, http://links.lww.com/MPA/A372). It is acknowledged that additional research is being conducted in these areas and that this document should not be considered an exhaustive review of all possible approaches.

\section{CASE FOR EARLY DETECTION: DEFINITIONS, DETECTION, SURVIVAL, AND CHALLENGES}

Recalcitrant cancers have been defined as those cancers that have 5-year survival rates of less than $50 \%{ }^{3}$ Among these, PC has the lowest 5-year survival rate of 6\%; others in the group of recalcitrant cancers (5-year survival) include lung (17\%), liver (18\%), esophagus $(19 \%)$, stomach $(29 \%)$, brain $(35 \%)$, ovary $(44 \%)$, and multiple myeloma $(45 \%))^{3}$ Pancreatic cancer incidence and death rates appear to be untouched by advances in science and technology. While surgery offers the only hope of 5-year survival in PC, fewer than $20 \%$ of patients are eligible for surgical resection, as the disease is already at an advanced stage at diagnosis. ${ }^{4}$ Treatment of locally advanced or metastatic PCs very rarely provides long-term survival. Meanwhile, the annual number of new PC cases in the United States is estimated to double (from 43,000 to 88,000 ) between 2010 and 2030 , and the adjusted number of deaths due to PC in that time period is estimated to increase from 36,888 to $63,000 .{ }^{1}$ Because of rising incidence and poor survival, $\mathrm{PC}$ is estimated to become the second leading cause of cancer deaths in the United States by $2020 .{ }^{1}$ Rahib et al ${ }^{1}$ have recently provided an in-depth study of future scenarios of cancer incidence and death rates, including those of PC.

Detection at an earlier stage and development of effective therapies are the cornerstones of reducing cancer death rates. In addition, by controlling the causative agent, the risk of cancers due directly or indirectly to infectious agents (eg, human papillomavirus, Helicobacter pylori, and hepatitis $\mathrm{C}$ virus) and those caused by environmental exposure (eg, smoking) can potentially be lowered. Thus, in most cancer subtypes, the cancer-related deaths have been decreasing. Compared with the number of cancer deaths in $2010(589,902)$, the projected number of cancer deaths in 2030 will increase by $\sim 30,000$ (to 620,000) despite an increase in incidence in total number of cancers in these 2 decades by $566,000 .{ }^{1}$ Colorectal cancer is estimated to drop from the top 4 in incidence and top 2 in deaths, due primarily to advances in colorectal cancer screening. Screening colonoscopy was introduced in 1997, and its acceptance as a screening test has been steadily increasing in the past decade; this has resulted in both decrease in incidence and decrease in mortality from colorectal cancer. ${ }^{5}$

Radical improvement in 5-year survival for PC will require concurrent advances in early detection to improve resectability rates and the development of chemotherapeutic agents to prolong survival following resection. Earlier detection would allow the downstaging of locally unresectable disease to allow resection, potentially reducing the risk of metastatic disease.

\section{Targetable "Early" Lesions for Early Detection of Pancreatic Cancer}

\section{Stages in the Progression From Precursor Lesions to Symptomatic PC}

Pancreatic intraepithelial neoplasia (PanIN), recognized as the precursor lesion for PC, progresses from PanIN-1 through PanIN-3 (PanIN-3 is synonymous with carcinoma in situ in the American Joint Commission for Cancer TNM classification) before becoming invasive. Pancreatic intraepithelial neoplasia 1 is considered hyperplastic and benign. Pancreatic intraepithelial neoplasia 2 is low-grade dysplasia, and PanIN-3 is high-grade dysplasia or carcinoma in situ. Pancreatic intraepithelial neoplasia 3 transitions to PC when the cancerous ductal cells that move through the basement membrane become invasive into the adjacent pancreatic parenchyma. Invasive PC can be classified by size; size of cancer generally correlates with stage of PC. Minute PC $(<10 \mathrm{~mm})$ is very often stage $1 \mathrm{~A}^{7}{ }^{7}$ but $\mathrm{PC}$ is rarely identified at this stage. Small PCs $(<20 \mathrm{~mm}, \mathrm{~T} 1$ in the TNM classification) form $10 \%$ of diagnosed PC and are metastatic in $45 \%{ }^{8}$ Nearly $90 \%$ of PC are diagnosed when they are large $(>20 \mathrm{~mm}$, T2 in the TNM classification); by then, they often have local extrapancreatic spread without (T3) or with (T4) involvement of major adjacent blood vessels. Stage I lesions are confined to the pancreas and may be small (stage 1A) or large (stage IB). Stage II lesions have extended beyond the pancreas without (stage IIA) or with (stage IIB) lymph node involvement. Stage III lesions involve adjacent major blood vessels, which could render them borderline resectable or unresectable. Stage IV cancers have distant metastases (M1) and are not resected regardless of feasibility of local resection of the cancer.

\section{Resectable PC}

Technical feasibility of complete surgical removal of all demonstrable PC ("resection with intent to cure") generally provides survival benefit but, in the majority, does not translate into longterm (5-year) survival and is rarely curative. Stages I and II are resectable, and some stage III lesions are considered resectable. Some stage III PCs are considered borderline resectable. The definition of borderline resectable varies ${ }^{9}$ and is often simply based on anatomic considerations that would predict high rates of margin positivity after resection. However, aggressive tumor biology and/or patient's physical inability to undergo both surgery and perioperative chemoradiation therapy could also negate any survival benefit of $\mathrm{PC}$ resection. Many borderline resectable cancers may benefit from neoadjuvant therapy prior to resection. Cancers with major vascular involvement are considered locally unresectable (stage III), and those with distant metastases are not considered for resection (stage IV). At diagnosis, most PCs are locally unresectable because of involvement of major blood vessels (stage III) or have distant metastases (stage IV). Only $20 \%$ of PCs are eligible for resection, and the average size of a resected PC is $30 \mathrm{~mm}^{10}$

\section{Defining "Early" PC}

At present, challenges remain in terms of defining what constitutes the earliest actionable lesion in the multistep progression of PC. There is little doubt that a PC with any degree of invasion, however small, is biologically advanced because both preclinical studies in autochthonous models and longitudinal studies on recurrence rates in patients with curative intent resection demonstrate the propensity for systemic micrometastases. Nonetheless, there should be a concerted attempt to identify a substantially larger proportion of patients with tumors that are resectable (currently only $\sim 20 \%$ of tumors are deemed resectable), because even 
with the potential for micrometastatic disease, the survival for patients with surgical resection is unequivocally better than that for those who cannot undergo surgery. As previously stated, even within the resected subset, the size of the primary lesion has a correlation with long-term survival, reiterating the need for "turning back the clock" as far back as possible in the diagnostic timeline. The decision matrix is less clear when one enters the realm of noninvasive precursor lesions. For example, autopsy studies have shown that $16 \%$ to $80 \%$ of adult pancreata harbor a PanIN, suggesting that this earliest precursor lesion is quite common and in the overwhelming majority of instances does not progress. ${ }^{11-13}$

\section{Molecular Alterations on the Path to Progression From PanIN-1 to Invasive Cancer}

There is a progressive accumulation of somatic genetic and epigenetic alterations as normal epithelium changes to low-grade PanIN-1, PanIN-2, and then to high-grade PanIN-3 and invasive cancer. ${ }^{14}$ While telomere shortening and mutations in KRAS appear to be early events in transformation of normal epithelium to PanIN-1, mutations in TP53 and SMAD4 are late events in progression of PanIN from 1 through 3. ${ }^{14}$ Although frequency of the TP53 and SMAD4 is higher in invasive cancer versus PanIN-3, mutations unique to invasive cancer are yet to be identified. Specific and sensitive biomarkers of PanIN-3 and early invasive cancer would be invaluable for detection of early PC.

\section{Timeline of Progression PanIN-1 to Large Invasive Cancer: Window of Opportunity for Early Detection of PC}

The timeline for progression from low-grade precursor lesions to invasive cancer is difficult to assess in longitudinal studies. Recent exome sequencing analyses ${ }^{15}$ of tumors from PC patients who have undergone a "warm" autopsy have shown that the genetic timeline for progression of this disease from initiation of the malignant "clone" to metastatic disease is nearly 2 decades, suggesting a wide window of opportunity for early detection, which has not been exploited. However, it is not known what proportions of this interval are at the stage of low-grade PanIN, highgrade PanIN, or invasive carcinoma.

To understand the timeline of progression of PC from resectable to unresectable disease, 114 computed tomography (CT) scans in 45 patients done at and prior to diagnosis of PC were retrospectively reviewed. ${ }^{16,17}$ These studies noted that onset of symptoms in PC coincided with development of signs of unresectability on $\mathrm{CT}^{16,17}$ On the other hand, PC was undetectable or resectable more than 6 months prior to its diagnosis, at a time when nearly all patients were asymptomatic. ${ }^{16,17}$ These studies suggest that if PC is detected before onset of cancerspecific symptoms, it will more likely be resectable. In another study, ${ }^{8}$ all 99 small $(<20 \mathrm{~mm})$ PCs were resectable, further suggesting that early detection of small tumors will improve resectability of PC. The finding that PC was undetectable by CT scan in a subset of patients 6 months prior their diagnosis underscores that CT scanning is not a good method for the earlier detection of PC; for example, there is a lack of sensitivity in identifying small cancers.

\section{Summary}

Currently, most PC is diagnosed when it is stage IIIB or IV (unresectable). Lesions with PanIN-3 (carcinoma in situ), invasive cancer confined to the pancreas (stage I), and resectable PC (stage II/some stage III) can be considered "early," as they are all resectable and have distinct histologic and molecular alterations compared with PanIN-1. As discussed in the following section, survival following resection even in "early" lesions varies widely, depending on presence and extent of invasion.

\section{Survival in Pancreatic Cancer}

\section{Impact of Early Detection and Treatment}

Cancer-specific symptoms predict early death as PC progresses rapidly once symptomatic; PC is diagnosed a median of 2 months after onset of symptoms, ${ }^{18}$ and death occurs 4 to 6 months following diagnosis. A delay in diagnosis is not uncommon because of the nonspecific symptoms and the expense of obtaining a CT scan of the abdomen, the test that is usually used for the initial diagnosis of PC. Delay to diagnosis is a significant independent prognostic factor for survival when stage is accounted for in multivariate analysis. ${ }^{19}$ In general, late presentation and poor prognosis are familiar characteristics of PC, which are illustrated by stagespecific survival curves for histologically confirmed PC derived from the National Cancer Institute (NCI) Surveillance, Epidemiology, and End Results (SEER) database for the years 2004 to 2010 (Fig. 1). Of 33,158 reported PC cases in this database, only $6 \%$ of patients presented with early, stage 1 disease, whereas more than $55 \%$ presented with distant metastasis, or stage IV disease. This suggests that the vast majority of cases progress asymptomatically through the early stages while the tumor is small and potentially treatable for cure. However, even at the earliest stage where the tumor is confined to the pancreas and less than $2 \mathrm{~cm}$ in its largest dimension (stage IA), 5 -year survival is only about $40 \%$. Poor survival is often ascribed to the likelihood that metastatic disease emerges early in the natural history of the malignancy, which is why resectable PC is treated using systemic approaches. ${ }^{20}$ The possibility that metastases occur as an early event is supported by autopsy studies that show most patients with PC who previously underwent resection die of metastatic disease. ${ }^{10}$ Early metastasis may explain the dramatic difference in survival between stage IA and IB cases seen in the SEER data as well as the indication that, of the $40 \%$ of patients with stage IA case who survive at 5 years, $25 \%$ eventually die of the disease (Fig. 1). Thus far, only PanIN-3 (carcinoma in situ), which is a preinvasive lesion, has been routinely curable for PC.

\section{Impact of Surgical Resection on Survival in PC}

Today, little evidence exists to suggest that long-term survival can be achieved in the absence of resection of the primary tumor. Although widely believed, the expectation that resection alters the disease course has not been rigorously established by randomized trials. Analysis of SEER data done for this review

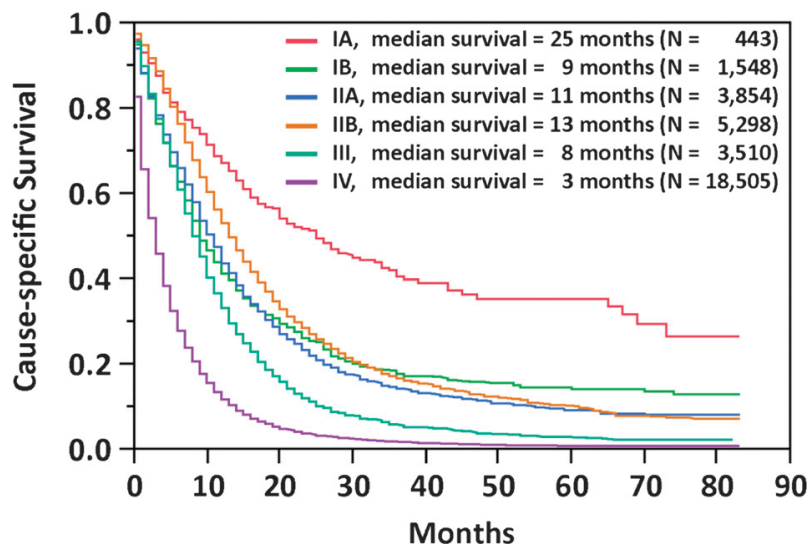

FIGURE 1. Stage-specific survival for histologically confirmed PC derived from SEER data. 
suggests a dramatic survival improvement for resected cases compared with those not operated on (Figs. 2A-C). Although this conclusion should be interpreted in light of the fact that resected cases will have had pathological staging, while the group of cases recommended for resection who refused will likely include cases with unrecognized advanced disease that would misleadingly reduce survival rates. Cox modeling of the SEER data for a typical stage 1B patient is shown in Figure 2C. Resection and radiation therapy appear to improve survival over no treatment. All 3 comparisons are likely affected by the difference in staging confidence between the pathologically staged resected groups and the clinically staged unresected groups. Eligible patients who do not undergo PC resection may live in rural communities where care is limited, may have been seen by health care providers who believe that PC is untreatable, or may be sicker individuals and therefore
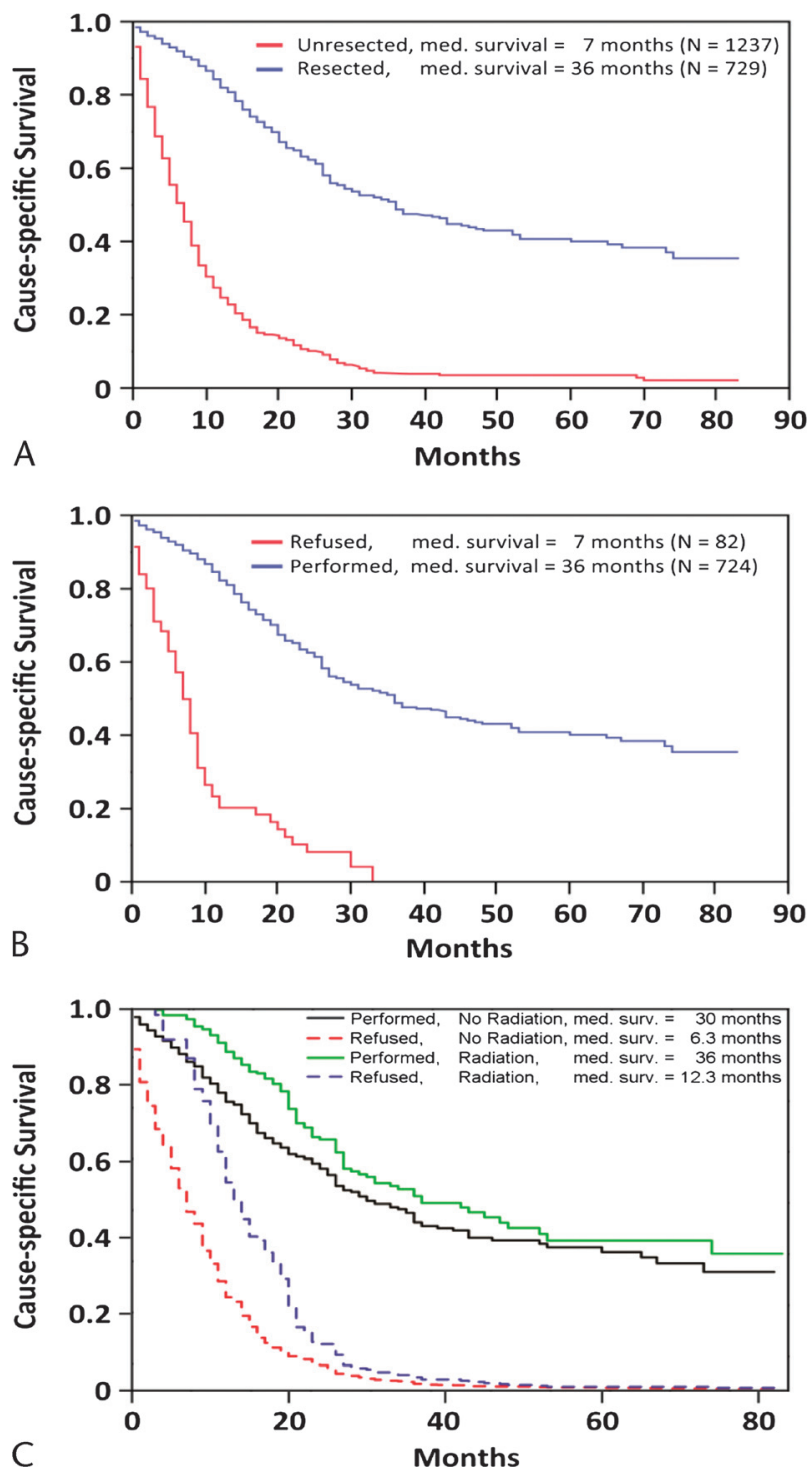

FIGURE 2. Effect of resection on survival in PC. A, The comparison for combined stages IA and IB cases. B, Survival comparison for cases (combined stages IA and IB) for which resection was recommended, and resection was either performed or refused. C, Predicted survival for a typical stage IB case (69-year-old woman) recommended for resection. surgery is not offered. In the latter case, there would be a selection bias for the unresected groups to have poorer survival. Although the SEER database does not include chemotherapy data, randomized controlled trials have been performed, including no treatment arms, suggesting that chemotherapy treatment improves survival from a median of 3 to 6 months to a median of 6 to 9 months.

In concert with the findings above, there is unequivocal evidence that diagnosis of $\mathrm{PC}$ at an earlier, resectable stage has a profoundly favorable impact on prognosis. The 5-year survival of resected PC is as high as $\sim 20 \%$ in large series major treatment centers, ${ }^{21}$ increasing to $30 \%$ to $60 \%$ for node-negative tumors of less than $2 \mathrm{~cm},{ }^{8}$ and as high as $60 \%$ for "minute" lesions less than $10 \mathrm{~mm} .{ }^{7}$ Even more dramatic are results in patients who are diagnosed with high-grade dysplastic, but still noninvasive precursor lesions such as a mucinous cystic neoplasm ${ }^{22}$ or intraductal papillary mucinous neoplasm (IPMN) of the pancreas. ${ }^{23,24}$ Surgery alone is essentially curative for these individuals with PanIN-3 or mucinous cysts with high-grade dysplasia, underscoring the critical importance of early detection. It is now well established that PC does not arise de novo but is rather preceded by noninvasive precursor lesions that undergo histologic and genetic progression culminating in invasive neoplasia.

\section{Potential Impact of Early Detection of PC on Survival}

For most types of cancer, the World Health Organization assumes that earlier detection would lead to a $30 \%$ greater cure rate (www.who.int/cancer/en/index.html). Whether this is also true for PC is a matter of debate. While developing effective strategies for treating metastatic disease will be important for increasing cure rates, there does appear to be an opportunity to increase survival rates by earlier detection and improved survival of early-stage disease. That tumor size is directly related to poor prognosis has been well established in the literature, ${ }^{10,25-28}$ and identification of tumors less than $1 \mathrm{~cm}$ in the largest dimension may further improve survival rates. ${ }^{29}$ Because stage IA patients have much better survival than later stages, one valuable strategy is to increase the percentage of individuals identified at stage IA.

Realistic modeling for an early detection screening program would include a gradual shift from the current stage distribution featuring late-stage disease to distributions with a higher representation of earlier stages. A more elaborate model was generated for this review using the 2004-2010 SEER data and determined stage distributions required to achieve a doubling, tripling, or quadrupling of average 5-year survival (Table 1). In this model, survival improvement is realized through both the increase in earlystage and the decrease in late-stage disease. These analyses are based on patient outcomes observed between the years 2004 (the earliest year that stage information was collected for the SEER program) and 2010. Survival during this time period was dependent on both the disease biology and the clinical interventions expected to modify survival deployed during the time period. As early detection screening is implemented, the realized survival rates may change because of increasing the percentage of patients with resectable disease and improvements in therapeutic interventions. The shift to earlier stages will also provide an opportunity to reevaluate our understanding of disease biology. For example, it is possible that a subset of PC cases progress so rapidly from undetectable disease to development of distant metastasis that the current level of stage IV cases remains largely unchanged.

\section{Summary}

An opportunity for improved survival through earlier detection of PC does exist. This conclusion is based on analysis of subjects with detectable PC. The apparent latent period between 
TABLE 1. Effect on 5-Year Survival by Changing the Relative Proportion Between Stages*

\begin{tabular}{lcccc}
\hline & Current Proportions & Doubling of Survival & Tripling of Survival & Quadrupling of Survival \\
\hline Stage & & & & \\
IA & 1.3 & 5.9 & 12.7 & 22.6 \\
IB & 4.7 & 13.4 & 21.8 & 28.8 \\
IIA & 11.6 & 22.0 & 26.6 & 26.2 \\
IIB & 16.0 & 19.9 & 17.9 & 13.1 \\
III & 10.6 & 8.7 & 5.8 & 3.2 \\
IV & 55.8 & 30.1 & 15.1 & 6.1 \\
Relative proportion parameter & 1.0 & 1.5 & 2.0 & 2.7 \\
Average 5-y survival & $4.1 \%$ & $8.2 \%$ & $12.3 \%$ & $16.4 \%$ \\
\hline
\end{tabular}

*A Cox proportional hazards model was used, with stratification for stage and sex, and a penalized spline with 4 degrees of freedom for age at diagnosis, and changing the relative proportion between paired, sequential stages.

development of detectable cancer and the development of metastatic disease suggests there is a window of opportunity for screening. Moreover, there may be an opportunity to provide inexpensive and accurate diagnostic tests, to reduce the delay to diagnosis in symptomatic patients. The median of 2 months between symptoms and diagnosis could be the difference between stages II and III disease. The size of the primary lesion appears to correlate with long-term survival, reiterating the need for diagnosis as early as possible in the tumor progression timeline, preferably to PanIN-3, which is a $100 \%$ curable lesion.

\section{Challenges and Biases in Early Detection of Pancreatic Cancer}

\section{Challenges}

\section{Early PC Lacks Clinical Symptoms}

Cancer-specific symptoms are associated with advanced PC (stages III and IV disease). Early symptoms are uncommon and nonspecific. Because symptoms occur late in the course of the disease, detection of resectable PC will require screening asymptomatic subjects.

\section{PC Is Relatively Rare}

Because of relatively low incidence of PC in the general population $(\sim 10 / 100,000)$, screening unselected populations for asymptomatic PC is not cost-effective with current technologies. Subjects 50 years or older display an age-adjusted incidence of PC of only 38 per $100,000 .{ }^{4}$ Even in this enriched cohort of older subjects, for every 100,000 subjects screened with an ideal biomarker ( $99 \%$ sensitive and 99\% specific for PC), 1000 subjects will be falsely identified as having PC. Nevertheless, while it remains cost-prohibitive and difficult with current technology to screen the general population for PC, models demonstrate screening of individuals younger than 70 years and who have a lifetime risk of PC of $16 \%$ or greater is cost-effective. ${ }^{30}$ There are a variety of factors that influence cancer risk. For example, $\sim 1 \%$ of adults older than 50 years who have new-onset diabetes will develop PC within 3 years. ${ }^{30}$ Family history of PC, environmental factors such as smoking, and certain genotypes can also influence risk. ${ }^{31-33}$ In practical terms, moderate-risk (5- to 10-fold increased risk) and high-risk (>10-fold increased risk) groups would likely benefit from early detection approaches. As methods are refined, imaging could also be applied to lower-risk groups, especially if combined with an inexpensive screening biomarker such as a blood test. In addition, the use of imaging agents to determine disease dissemination could be an important component of surgical decision making.

\section{Early PC Is Not Detectable by Routine Cross-sectional Imaging}

Computed tomography lacks sensitivity to detect small pancreatic lesions - not to mention precursor lesions such as PanINs. ${ }^{34,35}$ Therefore, based on available technology, detection of early lesions will require innovative noninvasive imaging or invasive testing, such as molecular endoscopic ultrasound (EUS).

\section{Abnormalities on EUS Are Not Specific for Lesions of Early PC \\ Endoscopic ultrasound has high resolution and detects le- sions as small as 1 to $2 \mathrm{~mm}$. Potentially EUS-guided fine-needle aspiration (FNA) biopsy can distinguish benign from malignant disease when focal lesions are present. ${ }^{34}$ However, in autopsy studies, a high proportion of individuals older than 50 years have significant changes in the pancreas due to age, obesity, smoking, alcohol, medications, and other environmental factors. ${ }^{36-38}$ These changes seen during imaging could obscure early lesions, espe- cially noninvasive PanIN-3. Hence, there is an unmet need to de- velop enhancements to EUS (eg, contrast or molecular EUS) or ancillary methods applied during EUS (pancreatic juice bio- markers) to distinguish true early lesions from false positives. ${ }^{39}$}

\section{Early PC Lacks Biomarkers}

There are no validated biomarkers of early PC. None of the available biomarkers possess a sufficiently high accuracy to be implemented for screening, even in high-risk patients. Carbohydrate antigen 19-9 (CA-19-9) is an oligosaccharide sialylated Lewis A antigen that is attached to mucin (MUC) core proteins, which has currently become the benchmark for following up patients with a PC diagnosis during treatment phases. Unfortunately, CA-19-9 may also be positive in patients with nonmalignant diseases including liver cirrhosis, chronic pancreatitis (CP), and cholangitis, as well as other gastrointestinal (GI) cancers. ${ }^{40}$ CA-19-9 discriminates between PC patients and control subjects with a sensitivity of $0.803(95 \%$ confidence interval, $0.777-0.826)$ and a specificity of 0.802 (95\% confidence interval, $0.780-0.823)$, respectively ${ }^{41}$ In 37 studies involving 1882 cases with $\mathrm{CP}$ as control subjects, the specificity of CA-19-9 was $82.8 \%{ }^{42}$

\section{Biases Inherent in Screening Programs}

Screening programs are subject to potential biases that complicate demonstration of benefit. ${ }^{42}$ 


\section{Lead-Time Bias}

This refers to the fact that detection of tumors via screening may seem to result in longer survival than control subjects (identified by clinical symptoms); however, the total time from malignant transformation to death may not have changed. Interventions that alter the natural history of disease (eg, potentially curative treatment) will improve survival rates in PC through early detection.

\section{Length Bias}

This refers to the potential for periodic screening programs to fail to identify rapidly developing tumors that have a short natural history until death and favor identification of less aggressive tumors with longer survival, thus exaggerating the survival benefit of the screening program. Our current understanding of disease progression suggests that an average of 11.7 years elapses from tumor initiation to overt cancer development, ${ }^{15}$ which affords some confidence that treatable, early-stage PC can be detected in annual or semiannual screening protocols. However, given the small sample size of the sequencing studies that provided this time estimate, the possibility for unrecognized, rapidly growing disease should be considered.

While the rapid progression of PC after the onset of symptoms could imply that screening programs of asymptomatic populations may detect only indolent tumors, it seems likely that this assumption is probably false. In the setting of FPC, an abnormal EUS, which can represent the development of PanIN-2 and 3 lesions, is seen in affected individuals many years before cancer develops. Some genetically susceptible but asymptomatic individuals have a completely normal EUS followed by increasingly abnormal EUS with subsequent histologic detection of PanIN-3 occurring over a period of 18 months to several years (T.A.B., unpublished data). This finding suggests that there can be progressive neoplastic change in some asymptomatic patients, but at the same time there is a window of opportunity for curative treatment prior to cancer onset. If a marker of PanIN-3 were available, such patients could be screened for high-grade lesions and offered therapy. It remains to be seen if similar strategies could be used in early detection of SPC.

\section{Selection Bias}

This refers to the propensity for self-selection under the assumption that only highly motivated subjects participate in screening programs. Because PC screening is a novel concept, considerable efforts will have to be invested in educational outreach to increase awareness about PC and need for early detection.

\section{False Positive "Pseudotumors" in PC Screening}

Heightened surveillance can lead to the overdiagnosis and overtreatment of tumors with indolent natural histories. This remains a concern, as removal of lesions requires major surgery. To mitigate this, approaches to finding actionable lesions need to have a very high degree of specificity.

\section{Summary}

There are a number of challenges to early detection. Based on the current technology, early detection of PC will require screening asymptomatic subjects from high-risk groups with invasive tests such as EUS. Defining high-risk groups that can be screened will require significant enrichment of the population in order to be cost-effective. Primary and secondary screens in potential high-risk groups will need to be highly accurate and inexpensive. Tools that can detect early lesions through noninvasive diagnostic screening tests and confirmatory molecular imaging will be the key to impactful changes in earlier detection.

\section{Defining High-Risk Groups for Pancreatic Cancer Groups at High Risk}

\section{Inherited Factors}

At present, less than $20 \%$ of FPCs can be attributed to a known genetic syndrome, ${ }^{2}$ including hereditary cancer syndromes and syndromes associated with inherited pancreatitis. The most commonly identified heritable PC syndromes include PeutzJeghers syndrome, familial atypical multiple mole melanoma, hereditary breast-ovarian cancer (including $B R C A 1$ and $B R A C A 2$ mutations), hereditary nonpolyposis colorectal carcinoma, and familial adenomatous polyposis. These syndromes convey a broad range of relative risk of developing PC; individuals with known familial adenomatous polyposis, BRCA1, or BRCA2 have less than $5 \%$ increased relative risk ${ }^{32}$ compared with the general population, whereas the risk in FAMM (lifetime risk 16\%) or PeutzJeghers (lifetime risk 36\%) is much greater. ${ }^{43}$ The number of affected first-degree relatives (FDRs) and environmental factors can further influence this genetic predisposition. There are families with PC affecting at least 2 FDRs and no associated genetic syndrome, currently defined as a "familial" PC kindred. ${ }^{44}$

Syndromes that lead to an increased risk of $\mathrm{PC}$ due to chronic inflammation of the pancreas are hereditary pancreatitis $(40 \%$ lifetime risk of PC by age 70 years) and, to a lesser extent, cystic fibrosis ( $<5 \%$ lifetime risk). The remaining $80 \%$ of cases with an inherited predisposition remain under the umbrella term of FPC, and susceptibility genes identification remains to be determined.

\section{New-Onset Diabetes}

When formally tested using oral glucose tolerance tests, nearly two-thirds of PC patients have diabetes mellitus (DM) at the time of diagnosis. ${ }^{45-48}$ In 2 complementary studies, the prevalence of DM was determined in a combined total of 642 PC subjects using fasting blood glucose and the American Diabetes Association criteria. ${ }^{49,50}$ In both studies, DM was present in nearly half the PC patients at diagnosis, ${ }^{49,50}$ and the majority $(75 \%-88 \%)$ of patients reported that the DM was of new onset, that is, diagnosed less than 24 months before diagnosis of PC. ${ }^{49,50}$ Conversely, other studies have shown that subjects with new-onset DM have a higher than expected likelihood of having PC. ${ }^{51-53}$ A population-based study reported that, compared with the general population, the cohort of subjects with late-onset ( $>50$ years) DM was 8 times more likely to be diagnosed with $\mathrm{PC}$ within 3 years of meeting criteria for DM. ${ }^{51}$ Other studies that analyzed subsets of subjects with recently diagnosed DM found an even higher prevalence of PC $(5.2 \%-13.6 \%){ }^{52,53}$ In a recent study, it was found that at the time of onset of DM, the cancer is often resectable. ${ }^{16}$ Thus, new-onset DM in subjects older than 50 years can act as a high-risk group for PC and can be the first "sieve" to enrich the general population for PC. This is the only highrisk group currently defined that targets SPC. The identification of new adult-onset diabetes as a PC risk factor significantly increases the applicability of screening methods. However, even this group will need to have an initial screening test with extremely high specificity and a secondary imaging screen that is accurate and inexpensive in order to make screening cost-effective. The ability to distinguish type 2 DM from PC-related DM (termed type $3 \mathrm{C}$ diabetes) would greatly facilitate the earlier detection of $\mathrm{PC}$ in this cohort.

\section{Other Epidemiological Risk Factors}

These have modest effect on PC risk, or the number subjects in the risk group is too large to subject to screening, but they could 
be included in risk models as risk modifiers. These include the following:

Smoking: Cigarette smoking is the best known factor for developing PC, increasing PC risk 1.5- to 3-fold. ${ }^{54}$

Age: Incidence of PC increases with age. ${ }^{55}$ More than threequarters of patients are diagnosed between 60 and 80 years; it is rare at younger than 50 years. It is important, however, to consider advanced age in the screening algorithm, as there is little point in screening individuals who have competing causes of death.

Racelethnicity: PC is more common in the African American population compared with the white population, some which may be attributed to socioeconomic factors and to cigarette smoking. ${ }^{55}$ Pancreatic cancer is disproportionally more common in those of Jewish heritage than in the rest of the population.

Gender: Cancer of the pancreas is only slightly more common in men than in women. Screening should target both men and women equally.

Chronic pancreatitis: Chronic pancreatitis is associated with increased risk of $\mathrm{PC}^{56}$ especially in those who smoke or have $\mathrm{DM}$. Longer duration of CP as seen in early-onset CP associated with hereditary pancreatitis or cystic fibrosis is associated with increased risk PC.

Longstanding diabetes: The relationship between DM and PC is complex. ${ }^{57}$ While longstanding DM is a modest risk factor for PC (relative risk, 1.5), new adult-onset DM can be a manifestation of PC. ${ }^{58}$ In addition, antidiabetic drugs may modify the risk of $\mathrm{PC}$ in $\mathrm{DM}^{59}$ From a screening perspective, longstanding DM, like smoking, is far too prevalent in the general population to be considered a high-risk group.

Partial gastrectomy may increase risk for PC. ${ }^{55}$

Dietary risk factors for PC include high meat intake, fried foods, and nitrosamines; fruits and vegetables may be protective against developing PC. ${ }^{55}$

ABO blood groups: Individuals with non-O-type blood are at modestly increased risk. ${ }^{60}$

\section{Summary}

Because symptoms occur at a very advanced stage of this cancer, PC has a poor prognosis. While detection and resection of high-grade PanIN-3 and early invasive PC will significantly improve survival, identifying such lesions will require screening of asymptomatic subjects for a lesion that is not visible on conventional cross-sectional imaging studies. To make progress in early detection of SPC, high-risk groups that will benefit from screening need to be defined. In addition, biochemical, molecular, or noninvasive imaging techniques capable of detecting high-grade PanIN-3 and early invasive lesion need to be discovered and validated. Subjects with a strong family history of PC have been offered screening for early PC using noninvasive and invasive imaging tests, which is discussed in an addendum to this article entitled Screening in Familial Pancreatic Cancer (Supplemental Digital Content 1, http://links.lww.com/MPA/A372).

\section{BIOMARKERS FOR EARLY DETECTION OF PANCREATIC CANCER}

\section{Overview}

Clearly the most logical time to identify PC is prior to invasion. Thus, given the lack of effective systemic therapies, developing biomarkers that could identify advanced precancerous lesions (PanIN-3, IPMN with high-grade dysplasia, or carcinoma in situ) will have the greatest impact on survival.

\section{Early Detection Biomarker: Target Population}

Currently, there is great need for the development of noninvasive but highly discriminatory biomarkers for early detection of PC. The first likely impact will be on those patients in whom clinically suspected PC would be part of the differential diagnosis. If a highly accurate and inexpensive biomarker was available, a simple blood test could be performed for patients with the common, but nonspecific symptoms associated with PC. These include epigastric pain, pain with eating, weight loss, unexplained diarrhea, and elevated bilirubin. Currently, the median time for diagnosis of PC is 2 months after presentation to the care provider. In part, this is because most clinicians do not want to order an expensive CT scan that is associated with radiation exposure for nonspecific symptoms, because the pretest probability remains relatively low. The hurdle to ordering the diagnostic test early in the assessment will be overcome, if the test is highly accurate and inexpensive. By ordering such a test in the beginning stages of the diagnostic workup, the delay to diagnosis could be considerably decreased, and it may be possible to downstage the disease.

A second impact for an accurate and inexpensive biomarker would be for patients with a strong clinical and radiological suspicion of PC; having such a tool may, in selected settings, obviate the need for more invasive studies such as tissue biopsy. Not only would this potentially save costs, but it would also expedite patients' workups in settings where tissue biopsies are difficult to obtain or where surgical intervention for the patient is limited. Conversely, for patients with a low suspicion of having PC on clinical presentation and radiological imaging, the value of having a highly discriminatory, noninvasive test could result in curtailing additional costly workup, while simultaneously reassuring the patient that they do not have a more serious condition and preventing the possibility of unnecessary intervention.

The last area of impact involves those patients with a higher risk for developing PC. These could include adult-onset diabetics or patients with a genetic susceptibility, who are in need of longterm surveillance. Currently, most screening strategies include invasive studies such as EUS and magnetic or endoscopic pancreatography for patients who have a relatively high risk of PC (lifetime risk $10 \%-15 \%$ or greater). However, there is an intermediate group of patients who have a lifetime risk of $\mathrm{PC}$ on the order of $1 \%$ to $5 \%$. These individuals in particular would not be candidates for expensive and/or invasive imaging tests, such as EUS, but would benefit from a highly specific biomarker that was relatively inexpensive. Thus, there are multiple settings where having a safe, highly discriminatory, noninvasive tool to detect PC would provide a mechanism for these patients to be screened or diagnosed safely and effectively, with the hope of decreasing mortality from PC through earlier detection.

To this end, there is an imperative need for innovation that will deliver accurate and affordable biomarkers that can detect preinvasive carcinoma in situ, as well as, early invasive disease.

\section{Characteristics of the Ideal Biomarker}

Ideal screening markers would be universally present in advanced preinvasive cancer and curable-stage $\mathrm{PC}$, while absent in individuals who are cancer-free or who have PanIN-1. These biomarkers should be readily detectable in easily obtainable biosamples, such as in body fluids (blood, saliva, stool) or other distant media (pancreatic fluid). To encourage compliance and use, the assays should be rapid, inexpensive, widely distributable to maximize test access, and practical to perform. Most importantly, to be effective as a screening tool, they must be highly sensitive and specific to accurately detect the critical target. 
The ideal markers would be the molecular alterations that accompany the evolution from incipient cancer (preinvasive PanIN-3 and/or IPMN with high-grade dysplasia) to early cancer that result from exfoliated cells or secreted markers. These include overexpressed and underexpressed RNAs, mutations and other genetic derangements important in the biological progression, and various epigenetic changes, especially those involving aberrant methylation. Other markers may represent unique expression of secreted MUCs and/or glycosylated and unglycosylated proteins. Most recently, the detection of circulating pancreatic cells (CPCs) may offer diagnostic, predictive, and prognostic markers.

\section{Biomarker Validation}

Currently, there are no clinically used biomarkers sensitive or specific enough for early detection. The dual goal of defining a clinically useful, early-detection biomarker is not only to identify the marker, but also to validate its purpose. Promising biomarkers should be validated by adhering to the PRoBE (Prospective Specimen Collection Retrospective Blinded Evaluation) criteria set forth by Pepe et al, ${ }^{61}$ when possible. This model consists of 5 phases:

Phase 1: Preclinical exploratory: to identify leads for potentially useful biomarkers and to prioritize identified leads.

Phase 2: Clinical assay and validation: to estimate the truepositive rate and false-positive rate for the clinical biomarker assay and to assess its ability to distinguish subjects with cancer from subjects without cancer.

Phase 3: Retrospective longitudinal: to evaluate the capacity of the biomarker to detect preclinical disease and to define criteria for a positive screening test.

Phase 4: Prospective screening: to determine operative characteristics of the biomarker-based screening test.

Phase 5: Cancer control: to estimate the reductions in cancer mortality afforded by the screening test.

As the candidate biomarkers for earlier detection are reviewed in following section, it is clear that the majority are still in the early phases of validation.

\section{Candidate Biomarkers}

\section{Methylated DNA Biomarkers}

Identification of biomarkers that accurately discriminate between early PC high-grade versus normal/low-grade lesions has been difficult. Recently, methylated DNA markers have been identified in PC. Ahlquist et $\mathrm{al}^{62}$ identified in a whole methylome discovery effort several methylated sequences that exhibit nearly perfect discrimination (area under the receiver operating characteristic curve between 0.9 and 1.0) at the tissue level for both PC and precancer, compared with normal pancreas. Several of these methylated DNA markers are in the process of being validated to discriminate high-grade from low-grade dysplasia. Unlike mutation markers that require unwieldy assay systems, single methylation markers or small panels of such markers are highly informative using simple assay methods.

Confirming the potential clinical value of these methylated genes as exfoliated markers, application of top candidates was tested in pancreatic juice and yielded high sensitivity and specificity for both PC and IPMNs that contained high-grade dysplasia. ${ }^{63,64}$ Detection rates by several methylation markers showed high discrimination of target neoplasms with low background levels in normal or CP control subjects. A nonoptimized marker panel detected $88 \%$ of $\mathrm{PC}$ at $90 \%$ specificity. Although these promising biomarkers still require optimization and multicenter validation, these observations demonstrate the high discriminant potential of markers exfoliated into the GI lumen and provide further biological rationale for a stool test application.

\section{Stool Testing: A Door to Population Screening}

Stool DNA testing represents an intriguing potential approach to general screening for PC. Based on the rational biology of tumor exfoliation and fecal recovery of signature DNA alterations, this approach has been fully validated for detection of colorectal neoplasia in recently reported studies. ${ }^{62,65,66}$ In early studies using nonideal DNA markers and nonoptimized assay methods, ${ }^{67,68}$ they found that it is possible to detect both early-stage PC and large IPMNs by stool DNA assay. Studies found that the copy number of BMP3, an early methylation marker candidate, is significantly and substantially higher from patients with PC than from control subjects. ${ }^{67}$ However, further research and test developments are necessary to achieve optimal detection performance. For example, because exfoliated DNA is broken down by luminal digestive enzymes during gut transit, shorter DNA fragments must be targeted to maximize the sensitivity of stool DNA testing for detection of upper GI neoplasms. ${ }^{69}$

Using stool DNA testing, it is possible to detect tumors from all GI sites using pan-GI cancer screening as a future approach to expand the value of stool DNA testing. Markers capable of tumor site predication would be desirable in a pan-GI application to help direct the diagnostic evaluation of a positive test result. Thus, a single noninvasive stool test could be used to efficiently screen the entire GI tract. Aggregate prevalence rather than individual site prevalence would be the key and would help to justify population screening of the less common, noncolonic neoplasms. Early data suggest that panels of methylated DNA markers can indeed be derived that accurately discriminate upper GI from lower GI tumors. ${ }^{70}$

\section{Salivary Biomarkers}

Saliva is an emerging biofluid poised for translational and clinical applications. In 2002, the National Institute of Dental \& Craniofacial Research made a significant investment toward developing the science as well as the translational and clinical utility of saliva. Four diagnostic biomarker categories are now known to be present in saliva. They include the proteome, ${ }^{71,72}$ the transcriptome, ${ }^{73-76}$ micro-RNA, ${ }^{77}$ and the metabolome. ${ }^{78}$ The University of California Los Angeles (UCLA) laboratory is 1 of 3 National Institute of Dental \& Craniofacial Research-funded groups that deciphered the salivary proteome and is responsible for the discovery and validation of the salivary transcriptome, micro-RNA, and metabolome. This group recently demonstrated the diagnostic utilities of salivary biomarkers for detection of PC. ${ }^{79,80}$

Tumors have been known to shed exosomes that shuttle tumor-specific constituents to different parts of the body, including salivary glands. The interplay between exosomes and salivary gland parenchymal cells leads to the development of tumorspecific constituents/biomarkers in the saliva of tumor-bearing patients. ${ }^{81}$ Recently, salivary extracellular RNA biomarkers have been used to confirm the diagnosis of PC

Using the PRoBE design, 12 transcriptomic salivary biomarkers were identified and validated. In a prevalidation study, a combination of 4 mRNA biomarkers (KRAC, MBD3L2, ACRV1, and DPM1) were able to differentiate PC patients from noncancer patients (CP patients and control subjects) with a sensitivity of $90 \%$ and specificity of $95 \%$. To obtain a realistic estimate of the predictive utility of the biomarkers, a leave-one-out crossvalidation was performed. The combined 4 PC salivary mRNAs model has a cross-validation error rate of 0.033 for distinguishing cancer and noncancer samples, achieving $96.7 \%$ (1\%-3.3\%) average prediction. This report provides the proof of the concept of 
salivary biomarkers for the noninvasive detection of $\mathrm{PC}$ and paves the way for a definitive validation study. ${ }^{80}$ The UCLA group is presently working to definitively validate salivary biomarkers that can accurately discriminate PC from non-PC patients. The hope is that these biomarkers may prove to be clinically impactful; however, it is acknowledged that the biomarker would have to have a near-perfect specificity in order to be cost-effective and that a positive test would require confirmatory imaging, such as EUS.

\section{Blood Biomarkers-Mucins as Diagnostic and Prognostic Markers}

Mucins are sugars that can be abnormally expressed through aberrant glycosylation during initiation and development of PC; MUCs have been explored as a target(s) for diagnosis or prognosis in both EUS-FNA, as well as blood-based biomarkers in various studies. ${ }^{82-85}$ Interestingly, a characteristic pattern of MUCs helps in the subtype classification and characterization of IPMNs. The IPMNs are classified into various subtypes, that is, intestinal $\left(\mathrm{MUC2}^{+}, \mathrm{MUC1}^{-}\right.$, and $\mathrm{MUC5} \mathrm{AC}^{+}$), pancreatobiliary (MUC2 ${ }^{-}$, $\mathrm{MUC1}^{+}$, and $\mathrm{MUC5AC}^{+}$), gastric (MUC2 ${ }^{-}, \mathrm{MUC1}^{-}$, and MUC5 $\mathrm{AC}^{+}$), and oncocytic (MUC1 ${ }^{+}, \mathrm{MUC2}^{-}$, and $\mathrm{MUC}^{-} \mathrm{AC}^{+}$) based on MUC expression. In EUS-based FNA studies, 73\% of ductal adenocarcinoma cases were found to be MUC7 positive. ${ }^{84}$ Interestingly, in the same study, $91 \%$ positive reactivity was observed for MUC4 on FNA from carcinoma cases. ${ }^{84}$ Notably, the immunohistochemistry of MUC4 and MUC16 on FNA was found to be quite useful in conjunction with cytology for atypical/ suspicious cases of PC. Retrospectively collected FNA specimens classified as adenocarcinoma, benign, and atypical/suspicious based on cytomorphologic criteria were further stained for MUC4 and MUC16; the MUC4 and MUC16 immunohistochemistry was $100 \%$ specific and $63 \%$ and $67 \%$ sensitive in differentiating benign cases from malignant. ${ }^{83}$ Although promising results have been observed in identifying various types of pancreatic masses in MUC-based FNA studies, further studies focusing on the accuracy of MUC staining for the diagnosis and differentiation of various pancreatic diseases should be carried out.

Mucin glycoproteins, because of their aberrant expression, also have high potential as diagnostic targets in blood. Among various MUCs, gel-forming MUC5AC, with de novo expression in precursor lesions of PC (PanIN-1), have been found to be highly sensitive and specific (Table 2). In the pursuit of detecting

TABLE 2. Mucin-Based Diagnostic and Prognostic Markers for Pancreatic Cancer*

\section{Target}

CA-19-9

Cancer antigen $50(\mathrm{CA}-50)$

Cancer antigen 19-5 (CA-19-5)

Cancer antigen 125 (CA-125/MUC16)

Cancer antigen $242(\mathrm{CA}-242)$

CAM17.1

MUC4

MUC5AC

DUPAN 2

PAM4

SPan-1

\section{Characteristics}

Carbohydrate reactive antibody epitope with SN (70\%-90\%) and SP (68\%-91\%) in serum, Food and Drug Administration-approved clinical prognostic biomarker

Limitations: elevated in benign diseases; Lewis $\mathrm{A}^{-} / \mathrm{B}^{-}$patients do not have this antigen; variable and poor $\mathrm{SN} / \mathrm{SP}$

Carbohydrate reactive antibody epitope with SN (78\%-84\%) and SP (70\%-85\%) in serum, diagnostic positivity similar to CA-19-9

Limitation: nonspecific elevation observed during jaundice and cholestasis

Carbohydrate reactive antibody epitope with SN (76\%-82\%), SP (73\%-85\%)

Limitation: performs poorly in comparison to CA-19-9

Transmembrane MUC with SN (45\%-57\%) and SP (76\%-78\%) in serum; $100 \%$ SP and $67 \%$ $\mathrm{SN}$ in FNAs

De novo expressed in very early precursor lesions, SN/SP varies as per pair of antibody used for screening

Limitations: nonspecific elevation in serum during liver cirrhosis, hepatitis, pancreatitis, and jaundice Undefined epitopic structure with SN (57\%-82\%) and SP (76\%-93\%) in serum

Limitations: low SN and SP due to its poor release into body fluids and nonspecific elevation during jaundice and cholestasis

Carbohydrate reactive antibody epitope with SN (67\%) and SP (90\%) in serum

Limitation: limited studies available on this antigen

Transmembrane MUC with SN (78\%-90\%) and SP (100\%) in FNAs

De novo expressed in very early precursor lesions

Limitations: low circulatory levels limit its performance in serum

Secretory MUC with SN (68\%-96\%) and SP (44\%-93\%) in serum (unpublished data)

De novo expressed in very early precursor lesions, potential diagnostic marker in combination with CA-19-9

Limitations: limited studies to date

Carbohydrate reactive antibody epitope with SN (48\%-64\%) and SP (85\%-94\%) in serum

Limitation: poor correlation of this marker is observed with CA-19-9

Carbohydrate reactive antibody epitope with SN (74\%) and SP (85\%) in serum, potential diagnostic marker in combination with CA-19-9

Limitations: limited studies are published

Carbohydrate reactive antibody epitope with SN (82\%-94\%) and SP (50\%-85\%) in serum, efficacy for diagnosis equivalent to CA-19-9, potential for predicting gemcitabine treatment failure

Limitations: limited studies performed

\footnotetext{
*For details, see Kaur et al. ${ }^{82-85}$

SN indicates sensitivity; SP, specificity.
} 
MUC carrier proteins, the meager amount of transmembrane MUCs in blood prevents their detection by conventional test platforms such as enzyme-linked immunosorbent assay or radioimmunoassay, which has restricted their exploitation as diagnostic targets. To circumvent these problems, attempts are being made to develop surface-enhanced Raman scattering-based immunoassays (100- to 1000 -fold more sensitive than conventional immunoassays) for the sensitive detection of MUCs. ${ }^{86,87}$

Apart from exploring MUCs themselves, autoantibodies formed against specific tumor-associated MUC antigens are prime candidates for exploiting their diagnostic potential. Pedersen et $\mathrm{al}^{88}$ utilized MUC1 and MUC4 glycopeptides to identify the autoantibody signature for colorectal cancer patients using prospectively collected clinical case-control sets as well as a blinded, nested, case-control study set. Although the MUC4based autoantibody signature failed to differentiate colorectal cancer cases from control subjects, the autoantibodies against MUC1 identified cancer cases with $95 \%$ specificity and $11 \%$ to $21 \%$ sensitivity. Interestingly, combination of MUC1-STn and MUC1Core3 data with data on autoantibodies to $\mathrm{p} 53$ peptides improved the sensitivities to $32 \%$ at $95 \%$ specificity. Overall, findings indicate that combining anti-MUC autoantibodies, which have good specificity, with other promising and sensitive biomarkers may improve accuracy for early diagnostic biomarkers for PC.

Mucins are differentially expressed tumor-associated antigens, and therefore they are attractive candidates for the early screening of PC. Future studies examining the glycan modification on specific carrier MUC, as well as their expression in EUS-FNA and circulating tumor cells (CTCs, could pave the way to improved diagnosis and prognosis in PC.

\section{Circulating Pancreas Epithelial Cells and Circulating Cell-Free Nucleic Acids}

The dissemination of pancreas cells in the setting of incipient PC (CPCs) or frank tumor cells into the circulation in the setting of known PC (CTCs) makes these circulating cells potential early biomarkers. Indeed, direct evidence for the early entry of precancerous pancreas cells into the circulation was borne from the studies of Rhim et $\mathrm{al},{ }^{89}$ in which fluorescently labeled, pancreas-derived cells could be identified in the circulation of mice genetically engineered to develop spontaneous PC, prior to the formation of overt tumors. ${ }^{89}$ Interestingly, CPCs could be found in circulation when pancreata contained only PanIN-2 and PanIN-3 lesions, even in the absence of invasive carcinoma on histology. Furthermore, recent data have shown that CPCs can be found in the bloodstream of patients with precancerous lesions of the pancreas and no diagnosis of cancer. In a recent publication, ${ }^{90} \mathrm{CPCs}$ were identified in the circulation of approximately $30 \%$ of patients with precancerous IPMNs and no known cancer. In addition, a portion of patients with BRCA mutations with no history of cancer or worrisome lesions on up-to-date screening protocols also had circulating epithelial cells (Saha et al, unpublished data). Thus, in both mouse models and humans, pancreatic ductal epithelial cells can enter into the bloodstream prior to the clinical diagnosis of cancer in patients at risk for the disease, and these cells are likely to be associated with the presence of advanced neoplasia. A portion of these CPCs may derive from a population of genetically abnormal cells in the pancreas that may represent the earliest forms of $\mathrm{PC}$, as subpopulations of CPCs have been found to contain PC-associated mutations in $K R A S$ and GNAS (A.D.R., verbal communication).

One of the advantages of using CPCs as a biomarker is that at least some of these cells can be collected via a simple blood draw. Circulating pancreatic cells have been shown to have a relatively high sensitivity ( $85 \%)$ and specificity (97\%) for identifying PC patients versus control subjects.

Currently, there are many methodologies to capture these CPCs. The most established technologies, and the ones currently in use in clinical trials, are microfluidic platforms utilizing "positive selection" in which epithelial cells are captured by antibodies specific to epithelial cell-specific epitopes, such as the epithelial cell adhesion molecule (EpCAM). At this time, positive selection capture techniques have the most data supporting their clinical use, and some versions of these devices have gone on to validation studies in clinical trials and CLIA (Clinical Laboratory Improvement Amendments) certification.

A potential disadvantage of this method of selection is that it excludes EpCAM-negative cells. Some reports suggest that CTCs, especially during advanced-stage cancer, may completely lose EpCAM expression, at least transcriptionally. However, recent comprehensive studies in which single CTCs were analyzed confirmed that the vast majority of cells do express EpCAM, even in the presence of a mesenchymal transcriptional program. Notably, positive selection platforms are able to capture CTCs with extremely low EpCAM surface protein expression, dramatically under the threshold needed to visualize such cells using amplified immunofluorescence techniques. Thus, it is unclear whether positive selection techniques truly "lose" clinically significant numbers of CTCs. To address this potential disadvantage, a number of groups have developed "label-free" microfluidic platforms to capture CPCs based on cell size and density after the depletion of red and white blood cells. Currently, these technologies suffer from low specificity with samples contaminated with high numbers of leukocytes. Other groups have sought to combine positive selection with label-free methods or to engineer automated cell selection methods after immunostaining whole-blood samples for epithelial markers. While these technologies are innovative and compelling, much work still needs to be done to show that these methods are sensitive and specific compared with positive selection platforms and to identify basic operating parameters that will allow for feasible transfer to CLIA-certified laboratories.

The most pressing issue with CPCs, as well as the other listed technologies, is whether they will be clinically informative. It is increasingly clear that the presence or number of CPCs alone will not have enough predictive power as a standalone test, as CPCs are identified in patients with precancerous IPMN, and these cells alone have not been predictive in determining those patients who have or who will develop cancer. Thus, additional analyses of these unique populations of pancreas cells are needed.

Many laboratories are developing new technologies to enable genetic and genomic analysis of small numbers of cells in a robust manner. Development of these technologies will not only inform studies of CPCs, but also enable analysis of cell-free circulating nucleic acids, such as circulating DNA. Indeed, the incorporation of next-generation polymerase chain reaction (PCR) technologies, such as picodroplet PCR and cold PCR, to reliably and sensitively identify single molecules of DNA containing PC-associated mutations, such as KRAS codon 12 mutations, among others, in CPCs could be valuable in developing a final clinical assay.

\section{Pancreatic Juice}

Current pancreatic screening protocols have the ability to identify pancreatic cystic lesions, many of which are IPMNs, but pancreatic imaging tests cannot reliably detect PanIN lesions, which is problematic because these lesions are considered the most common precursor to invasive PDAC. ${ }^{91}$ To improve the ability to evaluate the pancreas for evidence of pancreatic neoplasia in patients undergoing EUS evaluation, several studies have evaluated the utility of collecting pancreatic juice during an EUS 
evaluation and analyzing it for evidence of pancreatic neoplasia This approach utilizes knowledge of the genetic alterations in precursor lesions and the fact that most pancreatic precursor lesions arise within the ductal system. For example, it has been found that the prevalence of mutant GNAS in duodenal collections of secretin-stimulated pancreatic juice from patients with IPMNs is similar to that found in resected IPMNs, ${ }^{92,93}$ suggesting that pancreatic juice is a reliable sample for detecting molecular alterations in the pancreatic ductal system. Similarly, the detection of TP53 mutations in secretin-stimulated pancreatic juice samples was found to be a highly specific indicator of the presence of invasive $\mathrm{PC}$ or high-grade dysplasia. In contrast, KRAS mutations are commonly detected in the pancreatic juice samples of patients undergoing pancreatic screening, even subjects without any detectable evidence of pancreatic neoplasia by pancreatic imaging, (most of whom probably have PanIN-1 lesions), ${ }^{39}$ demonstrating that pancreatic juice sample analysis can provide information about the presence of pancreatic neoplasia that is complementary to the information provided by pancreatic imaging tests. ${ }^{39}$ The multicenter Cancer of the Pancreas Screening program ${ }^{94}$ is continuing to evaluate the diagnostic utility of pancreatic juice analysis and how best it can be incorporated into the evaluation of patients undergoing pancreatic screening.

\section{Innovating the Validation Phase}

Despite the identification of possible promising biomarkers that could assist in early detection, there remains no clinically useful test today. CA-19-9 remains the only available test in routine clinical use, and it is not used for diagnosis, but rather for therapeutic management. The progress toward blood tests for early detection has been hampered by a lack of suitable biospecimens that meet rigorous criteria for discovery and validation, due in no small part to the nature of the cancer itself. Second, in light of the challenges that are faced in studying PC, there is a need for innovative analytic design strategies.

The statistics of PC are well known. This translates into very low numbers of early-stage PC patients, which greatly explains why there are not adequate resources developed to construct sufficiently sized sample sets for early detection biomarker testing and validation studies. When the requirements of prospective consent, pretreatment quality samples, sufficient quantity, and stratification for covariates, such as comorbid disease, and so on, are added, the available qualifying samples drop dramatically.

In addition to developing and/or discovering biomarkers, there is a need to create collaborative programs for standardized patient sample procurement that would be suitable for validation studies. The rationale is that the detailed provenance of the samples makes them amenable to understanding biomarker assay performance in the patient/clinical setting. The samples need to be extremely well annotated (diagnosis, stage, DM status, etc). These annotated biological repositories need an organized system for sample distribution and statistical analysis. These biological repositories also need to make the methodological commitment to perform CA-19-9 on all samples as the criterion standard against which new biomarkers assays must exceed during discovery/ validation assays. Assays for these promising biomarkers should be performed blindly by the collaborator laboratories, and all statistical analyses of the returned data should be performed by the teams housing the annotated biorepository. From a validation design perspective, adherence to the PRoBE criteria set forth by Pepe et $\mathrm{al}^{61}$ for the Early Detection Research Network (EDRN) should be followed, with the notable exception of assays that cannot utilize banked specimens. An equally important next step is to modify the approaches for analyzing biomarkers. Two possible approaches include the incorporation of principles from adaptive design used in clinical trials and the incorporation of sequential analysis into biomarker validation.

\section{Summary}

Recent studies have been able to identify several promising biomarkers from saliva, stool, blood, and pancreatic juice. Clinical utility of these promising markers must now be validated through a standardized criteria set forth by the EDRN. The resources needed to validate these new biomarkers will include creation of annotated biorepositories with standardized real-time multimodal panels and new approaches to biomarker analysis.

\section{IMAGING EARLY PANCREATIC CANCER}

A recent NCI-sponsored think-tank on PC identified methods for early detection as among the most critical unmet needs in combating this disease. ${ }^{95}$ Their consensus view was that tools allowing earlier detection, patient stratification, and evaluation of therapeutic efficacy at earlier time points are of paramount importance. Not only could early detection provide a significant increase in patient survival, but also noninvasive imaging could enable appropriate treatment stratification and allow monitoring of therapeutic responses in vivo. Tumor size at diagnosis is the single most important prognostic indicator in PC. ${ }^{7,15-18}$ However, given the asymptomatic nature of early disease, resection is only possible in $20 \%$ of PC patients; often the detection of these tumors occurs incidentally upon surgical procedures or invasive imaging for other conditions. Indeed, PanIN progression to invasive PC occurs while lesions are still minute $(5-8 \mathrm{~mm})$, and extrapancreatic metastasis occurs remarkably early, with the majority of patients with lesions $10 \mathrm{~mm}$ or greater showing metastasis. ${ }^{8}$ No symptoms are observed, nor is detection by noninvasive methods typically possible until tumors approach the limits of resection. Current clinical imaging modalities can detect PDAC as small as $1 \mathrm{~cm}$ in some cases, but reliably closer to $2 \mathrm{~cm}$.

\section{Applications of Imaging for PC Detection-High-Risk Populations}

While it remains cost-prohibitive and difficult with current technology to screen the general population for PC, models demonstrate screening of individuals younger than 70 years and who have a lifetime risk of PC greater than or equal to $16 \%$ is costeffective. ${ }^{96}$ In this regard, it is important to note that despite its high mortality, the overall incidence of PC is relatively low; the overall lifetime risk is 1 in 80 (http://seer.cancer.gov/). However, there are a number of well-defined risk groups who are susceptible to PC that can significantly expand the utility of imaging for early detection. For example, $1 \%$ of adults older than 50 years who have new-onset diabetics will develop PC within 3 years. ${ }^{51}$

Family history of PC, environmental factors such as smoking, and certain genotypes can also influence risk. In practical terms, moderate (5- to 10-fold increased risk) and high-risk ( $>10$-fold increased risk) groups would likely benefit from imaging approaches. As methods are refined, imaging could also be applied to lower-risk groups, especially if combined with an inexpensive screening biomarker such as a blood test. In addition, the use of imaging agents to determine disease dissemination could be an important component of surgical decision making.

\section{Current Screening for Sporadic Cancers}

Patients who present with signs and symptoms that suggest a pancreatic neoplasm undergo initial noninvasive imaging with transabdominal ultrasound or CT that often reveals a pancreatic 
mass or fullness. The findings may represent an inflammatory process, benign entity, or malignancy. Endoscopic ultrasound provides unprecedented pancreatic imaging, tissue acquisition, and precise targeting of therapy and serves as an ideal minimally invasive image-guided system. Endoscopic ultrasound has been commonly performed since the early 1990s to help characterize pancreatic masses, to provide a tissue diagnosis, and to enhance staging. ${ }^{97,98}$ More recently, the utility of EUS in screening for PC has been evaluated in patients with a strong family history of $\mathrm{PC}$ as manifested by the presence of a high lifetime risk of PC. ${ }^{31,99}$ Based on current data, one may reasonably conclude that among high-risk patients CT detects only advanced-stage disease that provides minimal to no meaningful benefit to the patient. Likewise, EUS appears to identify many diminutive lesions of uncertain significance, leaving an unclear indication for operative intervention. While continued research is warranted, current strategies appear ineffective in meeting their intended goals. Given the controversy and unproven benefit of screening among high-risk individuals, one may question the rationale of screening for SPC given the even lower lifetime risk of $\mathrm{PC}$ within this cohort. Because of the lack of published data concerning the use of EUS in screening for PC, this document provides mostly opinion based on a review of data from trials conducted in high-risk patients and personal experience.

Pancreatic cancer develops in 3 settings including (1) SPC, which constitutes about $90 \%$ of patients; (2) FPC, associated with about $7 \%$ of cases; and (3) inherited cancer syndromes, which account for $3 \%$ of patients. ${ }^{2}$

When analyzing current screening data, it is important to clarify the specific patient cohort from which the data were obtained. Published screening studies included patients mostly from FPC kindreds (about 95\%) and few patients with inherited cancer syndrome $(\sim 5 \%)$. Patients with SPC were excluded because of their markedly disparate cancer risk, varying mechanisms of disease, clinical presentation, biological behavior, and imaging characteristics versus members of FPC kindreds. However, whereas some of these differences create even greater hurdles to screening for PC, others may offer an advantage.

\section{Anticipated EUS Experience}

Plausible expectations regarding the EUS findings encountered when screening for SPC include the following:

\section{Diagnostic Sensitivity}

The spatial resolution of EUS allows identification of diminutive structures below the limit of detection of any noninvasive imaging modality. For instance, with EUS, one can easily identify 1- to 5-mm or smaller structures including lymph nodes, bile ducts, pancreatic ducts, blood vessels, solid masses, and cystic masses. However, this is not to suggest that EUS may reliably detect all PCs and precancers of this size. In fact, tumors measuring greater than $1 \mathrm{~cm}$ are sometimes missed with EUS.

A number of factors impact the sensitivity of EUS for detecting diminutive lesions including the relative echo density and echo pattern of the target lesion (cancer and precancer) compared with the background structure (pancreas). For instance, a typical hyperechoic neuroendocrine tumor is usually easily detected in patients with a normal isoechoic salt-and-pepper-appearing pancreas. More problematic is the detection of hypoechoic and heterogeneous-appearing pancreatic carcinomas in the setting of CP. Other characteristics of the target lesion that impact their detection include the shape, border features, degree and uniformity of attenuation, and location within the pancreas. In addition, proximate and intervening pancreatic and even peripancreatic structures, such as pancreatic calcification, fibrosis, omentum, and small and large bowel, may hinder the detection of diminutive pancreatic masses.

\section{Diagnostic Specificity}

While the sensitivity of EUS for detecting diminutive pancreatic masses may prove suboptimal, likely more problematic is poor test specificity. One would anticipate difficultly discriminating diminutive PCs and precancers from normal background changes of the pancreas. Data from high-risk screening studies indicate the common presence of $\mathrm{CP}$-like features, cysts, and solid nodules among this patient cohort. Their presence leads to much uncertainty regarding the significance of any identified diminutive pancreatic lesion. In fact, there is often doubt as to whether the perceived diminutive mass represents any pathology or merely a variant of the underlying ductal and/or parenchymal changes. It remains to be determined whether these typical pancreatic alterations will be seen in patients with PC as found in high-risk kindreds. Patients who develop PC may also demonstrate other yet described pancreatic changes, thereby limiting the diagnostic sensitivity and specificity of the EUS examination. Furthermore, patients often demonstrate pancreatic fibrosis in the absence of endocrine or exocrine dysfunction. ${ }^{100}$ Such "asymptomatic" fibrosis has been reported in alcoholism, advanced age, male sex, obesity, and cigarette smoking. These features have been associated with altered pancreatic imaging and histology in patients without evidence of CP and complicate screening efforts.

\section{Interobserver Agreement and Operator Dependence}

One of the greatest limitations in the use of EUS is the relatively poor interobserver agreement (IOA) and the operator dependence. Wallace et $\mathrm{al}^{101,102}$ and Wallace and Hawes ${ }^{101,102}$ studied the interpretations from 11 expert endosonographers blinded to clinical information who evaluated videotaped examinations for the presence of CP features among 33 patients with CP. While agreement was good for 2 of the 9 features including duct dilatation $(\kappa=0.6)$ and lobularity $(\kappa=0.51)$, agreement was poor for the other 7 features $(\kappa<0.4)$. There was moderate overall agreement for the final diagnosis of $\mathrm{CP}(\kappa=0.45)$. Topazian et $\mathrm{al}^{103}$ assessed IOA for interpretation of EUS in persons at high risk for PC using recorded video clips and found similar results. In a study pending publication, unacceptable rates of agreement when evaluating solid hepatic masses were found. The issues of poor IOA and operator dependence are expected to limit the utility of EUS to the most experienced endosonographers.

\section{EUS-Guided Biopsy}

Just as the diagnostic sensitivity and specificity of EUS imaging must be considered, so too must the performance characteristics of EUS-guided biopsy. Several issues must be considered including the indications for biopsy. Given the poor specificity of EUS imaging, most will favor performing EUS-FNA even for resectable lesions. However, sampling diminutive lesions can be technically challenging when sampling in other settings (eg, <4-mm hepatic metastasis) negatively impacts diagnostic sensitivity. The decreased sensitivity results from difficulty targeting and accessing the lesion, the need to traverse a large volume of normal and nondiseased tissue, and the nominal distance the needle passes within a diminutive structure. The need to obtain the necessary 10 groups of malignant cells often requires a greater number of needle passes. As a result, the safety of additional passes within a relatively normal pancreas must be considered. In addition, limited data suggest the heightened risk of false-positive FNA in this setting given the difficulty of cytologically discerning PanINs and IPMNs from ductal carcinoma. Therefore, neither a positive nor negative cytologic interpretation 
can be viewed with absolute certainty, likely risking surgical resection for a benign lesion and sometimes inappropriate observation for an undiagnosed malignancy.

\section{Anticipated Needs and Areas of Investigation}

Anticipated needs to enhance the use of EUS in screening for SPC include the following:

\section{Newer EUS Technologies}

Emerging technologies may help to overcome the limitations of conventional EUS imaging. These technologies are at varying staging of development and study and include contrast-enhanced EUS, elastography and digital image analysis, as well as enhanced resolution for basic gray-scale imaging. Contrast-enhanced EUS is believed by some to improve differentiation of PC from $\mathrm{CP}^{104,105}$ Contrast enhancement evaluates the perfusion characteristics of the pancreas, that is, the degree of vascularity within a focal region. Chronic pancreatitis features, as opposed to PC, are defined as having no detectable vascularization before contrast injection, a regular appearance of vessels over a distance of at least $20 \mathrm{~mm}$ after injection of the contrast medium, and the presence of arterial and venous vessels following injection. ${ }^{104,105}$ Conversely, malignant lesions demonstrate no detectable vascularization with conventional power Doppler scanning, irregular appearance of arterial vessels over a short distance using contrast-enhanced technique, and no detection of venous vessels inside the lesion.

Elastography, or strain imaging, allows differentiation of tissues based on consistency. The main principle of strain imaging is that malignant tissue tends to be harder than benign tissue. Endoscopic ultrasound elastography can be accomplished real time using newer echoendoscopes with the images being represented in transparent color superimposed on the conventional grayscale B-mode scans. ${ }^{106}$ Limited studies have evaluated the role of EUS elastography in pancreatic disease. In 73 patients with a combination of normal pancreas, CP, and focal lesions, Janssen et $\mathrm{al}^{107}$ were able to distinguish between normal pancreas and $\mathrm{CP} /$ focal lesions. However, they found that $\mathrm{CP}$ and tumors could not be distinguished by elastography, probably because of their similar fibrous architecture. ${ }^{107}$ No other studies have specifically evaluated the ability to differentiate between normal pancreatic tissues and CP using elastography. Further study of its test characteristics, especially in comparison with EUS morphologic evaluation and direct pancreatic function testing, is in order before EUS elastography of the pancreas is put into routine clinical practice.

For these new technologies, published studies provide few data, and the techniques are not in widespread use. These technologies as currently used provide minimal or no advantage to skilled endosonographers and likely less utility for minute PCs; however, additional study is warranted.

\section{Novel Use of EUS-Acquired Tissue and Fluid}

Newly discovered molecular markers may be evaluated from specimens obtained at EUS to further enhance the diagnostic accuracy and provide theranostic potential. This information may serve as a useful filter (along with new-onset diabetes, weight loss, elevated CA-19-9, etc) to help select the ideal candidates to undergo screening and to guide the timing and intensity of screening efforts. The minimally invasive approach and finely targeted capability of EUS are likely to prove essential in this regard.

\section{Training and Education}

Although the issues pertaining to poor IOA and operator dependence may be partly overcome by training and teaching modules, prior efforts suggest a likely limited utility of such efforts.
Furthermore, most endosonographers still rely on radial imaging that, because of its inferior spatial resolution and subsequent need for a linear examination, will limit the detection of diminutive carcinomas. In addition, most endosonographers feel that the value of EUS for evaluating PCs is limited to obtaining a tissue diagnosis. They feel that there is no opportunity to enhance staging over CT alone believing that in their hands EUS too infrequently identifies meaningful and verifiable evidence of more advanced disease not detected by CT. This belief applies to the evaluation of symptomatic larger PCs. Given the beliefs and practice pattern around the world, one may assume that most endosonographers will feel there is even lesser role for screening for SPCs. Furthermore, the infrequent presence of PC in high-risk groups may limit the intensity and thoroughness of EUS screening examinations.

\section{Understanding the Ideal Targets}

Many of the questions concerning the utility of EUS cannot be answered until there is clearer understanding of the size threshold below which a PC must be detected to enable initial curative resection or tumor downstaging with resection, or at least a meaningful impact on survival. Otherwise, providing an earlier diagnosis and lead time bias without improving clinically important outcomes and the window of opportunity may close. A reasonable target may be precancerous lesions. However, at this time, there is no way to identify high-grade PanINs.

There is need to collect the necessary data to develop a clinically meaningful definition of early PC and to identify the clinical, imaging, laboratory, and pathological features to determine the potential utility of PC screening and to develop such programs. While hopefully such definitions and criteria can be established, there is the possibility that the PC tumor biology may prohibit any successful screening strategy.

\section{Understanding the Typical Appearance of a "Normal" Pancreas}

Patients who develop FPC and even unaffected family members typically demonstrate morphologic alterations in pancreatic appearance as previously discussed. There is an incomplete understanding as to the prevalence and timing of their development in such patients. It would be important to determine whether the same morphologic alterations also develop in patients who develop SPC or whether alternate changes occur. The timing of such changes is important as well. From retrospective review of CT scans done prior to PC diagnosis, Gangi et $\mathrm{al}^{17}$ determined that the majority of $\mathrm{PC}$ is resectable 6 months before clinical presentation, suggesting that early detection will lead to a higher proportion of PC being resected. While preliminary studies suggest an ideal timeline to begin screening for PC, a negative EUS and CT may not entirely exclude a developing $\mathrm{PC}$, and repeat imaging may be necessary to detect an occult cancer and to determine the presence and timing of any pancreatic background changes that herald a PC.

The advent of EUS was an important advance in the care of patients with pancreatic pathology. It is not clear that the same will hold true for screening for PC. Despite advances in EUS technology and training, there has been no meaningful progress over the past 20 years in the EUS diagnosis, biopsy, or staging of symptomatic larger pancreatic carcinomas. One must assume the challenge will be even greater when screening for diminutive PC.

\section{Novel Technologies: Could Molecular Imaging Be the Answer to Early Detection?}

Improvements in our understanding of molecular mechanisms of disease and imaging could lead to noninvasive methods 
for early detection of precancerous lesions and tumors of the pancreas. Diagnosing preinvasive or early-stage invasive PC remains problematic because imaging contrast is not sufficient to differentiate tumor from inflamed or normal pancreatic tissue using conventional imaging. In contrast, molecular imaging has the potential to visualize early-stage cancer at the molecular level before morphological-anatomical changes can be detected.

Molecular imaging allows visualization of biological processes at the molecular level. Imaging agents that interact with proteins that are uniquely expressed, overexpressed, mislocalized, or absent in tumor cells or their microenvironment could be used to distinguish tumors from surrounding tissues. These agents could provide a rapid, noninvasive mechanism for early detection and localization of pancreatic tumors, allowing earlier intervention or identification of metastatic disease for patients who have been identified as candidates for surgery. To be clinically relevant, molecular-based imaging agents must be highly specific, sensitive, and amenable to integration into current imaging modalities, including endoscopy. Some targeted imaging agents developed for visualizing pancreatic tumors are at the proof-of-concept stage, whereas others are ready for clinical studies.

\section{Imaging Tumor Vasculature}

A recent study suggests that the "angiogenic switch" can already be observed at precursor lesion stages of colon carcinoma. ${ }^{108}$ In an immunohistochemical analysis of 210 human surgical samples from the colon, Staton et al ${ }^{108}$ have shown that the "angiogenic switch" occurs at the onset of dysplasia at the adenoma stage of the adenoma carcinoma sequence of colon cancer. Similar findings may be true for precursor lesions of PC. Khorana et $\mathrm{al}^{109}$ have shown that expression of tissue factor can be observed in $77 \%$ of PanIN lesions from a total of 40 patients. In invasive PC, this tissue factor expression strongly correlated with vascular endothelial growth factor (VEGF) expression $(P<0.0001)$ and microvessel density $(P=0.01){ }^{109}$ Further studies are needed to study whether the "angiogenic switch" is also already present at the level of precursor lesions in PC analogous to colon cancer.

Because it is cost-effective, portable, noninvasive, and widely available, ultrasound is a modality that is used clinically and has been adapted for use in molecular imaging. By taking advantage of the physics of ultrasound, researchers have developed microspheres filled with gas (microbubbles) that can be sensitively detected. Because of their size, microbubbles are restricted to the vascular compartment. ${ }^{110}$ The VEGF receptor type 2 (VEGFR2) protein is critical for angiogenesis physiology and in many cancers including PC. Several steps are involved in tumor angiogenesis including proliferation, migration, and invasion of endothelial cells; formation of endothelial cells into tubular structures; maturation of blood vessels; and blood vessel degeneration. ${ }^{111}$ This complex process involves the coordination of several signal transduction pathways and is regulated by various proangiogenic and antiangiogenic factors. ${ }^{12-114}$ VEGFR2 (called $\mathrm{KDR}$ in humans) is one of the best-characterized molecular markers of tumor angiogenesis. It is an endothelium-specific receptor tyrosine kinase, and activation of the VEGF/VEGFR2 axis triggers multiple signaling networks that result in endothelial cell survival, mitogenesis, migration, differentiation, and vascular permeability. ${ }^{111}$ VEGFR2 is considered a major factor of tumor angiogenesis and is overexpressed on tumor endothelial cells of angiogenic vessels in PC in patients. ${ }^{111,115-123}$ These findings make VEGFR2 an attractive molecular target for imaging PC. Deshpande et $\mathrm{al}^{124}$ conjugated an anti-VEGFR2 antibody to microbubbles and were able to image PC xenografts.

Foygel et $\mathrm{al}^{125}$ recently identified a PC-specific endothelial marker absent in patients with $\mathrm{CP}$, thymocyte differentiation antigen 1 (Thy1). Using antibodies, they generated Thy1-targeted microbubbles that were able to detect PDAC and PanINs in genetically engineered mouse models ${ }^{125}$

\section{Tumor Epithelial Cells}

For decades, integrin molecules have been the center of interest in molecular imaging of many cancers. Integrins such as $\alpha_{v} \beta_{6}$ and $\alpha_{v} \beta_{3}$ are overexpressed in tumor compared with normal tissue. The selective expression of specific integrins is contributing to their relevance as potential targets for molecular imaging purpose.

The integrin $\alpha_{v} \beta_{6}$ is present only in the epithelium, but its levels are strongly increased in a number of tumor types and were found to be highest in PDAC compared with other GI adenocarcinomas. Levels of $\alpha_{v} \beta_{6}$ have been associated with patient outcomes. A peptide derived from the foot-and-mouth disease virus interacts, with high levels of affinity and specificity, with $\alpha_{v} \beta_{6}$. Hausner et al ${ }^{126,127}$ created an $\left[{ }^{18} \mathrm{~F}\right] \mathrm{FBA}$-coupled peptide, A20FMDV2, that detects $\alpha_{v} \beta_{6}$ on tumor cells. Positron emission tomography (PET) imaging demonstrated that tumor tissues took up 23-fold more $\left[{ }^{18} \mathrm{~F}\right] \mathrm{FBA}-$ PEG28-A20FMDV2 than healthy pancreatic tissue. ${ }^{128}$ Zhu et al ${ }^{129}$ have developed a single-photon emission CT-based $\alpha_{v} \beta_{6}$ integrinavid probe radiolabeled with technetium Tc $99 \mathrm{~m}\left({ }^{99 \mathrm{~m}} \mathrm{Tc}\right) .{ }^{99 \mathrm{~m}} \mathrm{Tc}$ SAAC-S02 has been designed using a novel class of $\alpha v \beta 6$ integrin small peptide-based ligand called knottins. ${ }^{130}$ One hour after injection in a murine model bearing lung (HCC4006) xenograft, ${ }^{99 \mathrm{~m}} \mathrm{Tc}-$ SAAC-S02 exhibited a tumor-to-muscle uptake ratio of $4.8: 1$ by tissue harvested and raised to $6.8: 1,6$ hours after injection. The tumor is visible on SPECT/CT images but no quantitative data are available.

Similarly, another group of investigators have been working on targeting $\alpha_{v} \beta_{6}$ integrin using a SPECT agent. Liu et al ${ }^{131}$ evaluated ${ }^{99 \mathrm{~m}}$ Tc-radiolabeled HYNIC-conjugated HK-denoted peptide agent $\left({ }^{99 \mathrm{~m}} \mathrm{Tc}-\mathrm{HHK}\right)$ in a murine model bearing BxPC-3 $\left(\alpha_{\mathrm{v}} \beta_{6}-\right.$ positive) tumor. Planar and tomographic $\gamma$ images obtained 1 hour after injection showed a tumor-to-blood and tumor-to-muscle uptake ratio of 2:1 by image quantification, and this was confirmed by $\gamma$-well counting. Comparing ${ }^{99 \mathrm{~m}} \mathrm{Tc}$-HHK with ${ }^{18} \mathrm{~F}$-FDG, the most commonly used radiotracer for cancer detection, the investigators demonstrated that ${ }^{99 \mathrm{~m}} \mathrm{Tc}-\mathrm{HHK}$ exhibited a positive signal only in mice bearing BxPC-3 ( $\alpha_{v} \beta_{6}$-positive), whereas ${ }^{18} \mathrm{FDG}$ displayed a positive signal in $\alpha_{v} \beta_{6}$-positive and $\alpha_{v} \beta_{6}$-negative.

$\alpha_{v} \beta_{3}$ Is an integrin that has been shown to be overexpressed in many tumor types, including pancreatic. Trajkovic-Arsic et $\mathrm{al}^{132}$ proposed a multimodal molecular imaging $\alpha_{\mathrm{v}} \beta_{3}$ integrin-targeted probe for in vivo detection of PC. The ${ }^{68} \mathrm{Ga}-\mathrm{NODAGA}-\mathrm{RGD}$ probe has been designed to be detected by magnetic resonance imaging (MRI), PET, and optical imaging. After ${ }^{68} \mathrm{Ga}-\mathrm{NODAGA}$ RGD injection in a murine model of spontaneous PC, PET images showed a clear uptake of the probe in PC region expressing $\alpha_{v} \beta_{3}$ integrin anatomically delineated by MRI. According to the authors, this strategy can further be exploited for identification of patients with $\alpha_{v} \beta_{3}$ PC, fluorescence-guided surgical removal of PC, and application of $\alpha_{\mathrm{v}} \beta_{3}$-targeted therapies.

\section{Plectin 1}

In normal cells, plectin 1 is a cytosolic protein with 11 isoforms that have different cellular localizations and functions. In PC and other cancers, plectin is aberrantly localized to the extracellular surface giving exquisite selectivity for cancer. ${ }^{133,134}$ Plectin 1 in cancer and a lead targeting peptide, PTP (plectin 1targeted peptide), were discovered through phage display-based functional proteomics and has recently been developed as a SPECT agent and used to identify tumors in orthotopic models of PC and metastasis. ${ }^{135}$ 


\section{Receptor Tyrosine Kinase Axl}

An additional strategy in the detection of PC consisted in imaging of the receptor tyrosine kinase Axl expression. It transduces signals from the extracellular matrix into the cytoplasm by binding growth factors and thus is involved in the stimulation of cell proliferation. Given its biology, Axl has been a drug target for pharmaceutical development. Because Axl is overexpressed in $55 \%$ of all PCs, it potentially would not be an appropriate early detection marker, but an Axl-molecularly targeted imaging agent may find relevance for patient treatment stratification. The investigators designed a ${ }^{125}$ I-radiolabeled monoclonal antibody Axl-targeted probe $\left({ }^{125} \mathrm{I}-\mathrm{Axl} \mathrm{mAb}\right)$ and evaluated its potential in a severe combined immunodeficient murine model harboring subcutaneous PC cell line xenografts CFPAC (Axl high) and Panc1 (Axl low). ${ }^{136}$ The SPECT/CT images exhibited a clear and specific accumulation of radioactivity in the CFPAC tumors by 24 hours, which could still be clearly visualized at 120 hours after ${ }^{125} \mathrm{I}$-Axl mAb injection. The data were confirmed by $\gamma$-well counting with a tumor-to-muscle uptake ratio of about 10:1 in CFPAC tumor and about 4:1 in Panc1 model. Unfortunately, the very high and long-lasting ${ }^{125} \mathrm{I}-\mathrm{Axl} \mathrm{mAb}$ activity in the blood could potentially limit clinical application.

\section{Bombesin Receptors and MUC4 MRI Approach}

An unusual but nevertheless interesting approach has been imagined by Montet et $\mathrm{al}^{137}$ using MRI modality. The investigators evaluated the feasibility to diagnose PC-targeting Bombesin receptors expressed in normal pancreatic tissue but not in PC. Using $\mathrm{BN}-\mathrm{CLIO}(\mathrm{Cy} 5.5)$ as a probe and the $\mathrm{T} 2$ relaxation of healthy pancreatic tissue causes it to appear dark, whereas tumors stay bright, allowing the authors to detect PC in mice.

\section{Summary}

Currently, because of the low incidence of PC and the limitations of current technologies, it is not feasible or cost-effective to screen for cases of sporadic cancer. There are patient populations already identified that have heightened risk, and they are currently being screened. With the advent of serum biomarkers, more of the population without family or genetic risk factors could be screened, which could result in the earlier diagnoses of sporadic cases. Finally, molecular imaging has emerged as a potential way to identify smaller lesions, translating into the potential to diagnose at a much earlier stage than is available. Molecular imaging has the benefit of being able to identify differences between tumor and normal or CP on a molecular level, not based on morphological differences. Being able to combine molecular imaging with conventional imaging (ie, molecular ultrasound, fluorescence endoscopy, or PET/MRI) could have important implications for patient outcomes.

\section{COLLABORATIVE STUDIES}

\section{Overview}

Collaborative research often occurs when 2 or more parties perceive that completion or acceleration of a research project requires additional expertise, technology, samples, patients, model systems, or other components. In some cases, collaboration results from scientific relationships, friendships, or proximity. Modern research in general and especially research in PC are usually enhanced by collaborative research or team science. Unfortunately, there are many barriers to collaborative research. Barriers include the "silo" nature of many studies resulting from competition, fear of intellectual theft, the structure of the systems of rewards in research, lack of access to resources or samples, institutional constraints, lack of collegiality, poor leadership, and lack of experience with effective team research programs.

\section{State of the Art}

There are several broad categories of resources that are required for research in PC: model systems (cell lines, animal models, in vitro systems, in silico systems), patient resources (patient information, clinical samples, clinical trials), research capability (instrumentation, laboratory skills and technology, expertise, creativity, workforce), funding, and incentives. Most of these areas, with the exception of funding and incentives, have seen progress in the past 20 years. There are now improved model systems, patient resources, and research capability.

\section{Patient Populations}

For the purposes of this article, patient resources for biomarkers will be categorized into 6 classes: the general population, an at-risk population, symptomatic patients who do not have PC, patients with newly diagnosed disease, patients undergoing treatment, and patients who fail treatment.

- General population resources include data and sample collections from the population at large and may be collected from population-based studies, clinic based (primary care), or convenience sampling (random volunteers). These are typically regarded as "healthy controls."

- The at-risk population includes unaffected relatives from families with a genetic predisposition to cancer and unaffected individuals whose lifestyle, exposure to carcinogens, or presence of ancillary disease (ie, pancreatitis, diabetes) puts them at risk for developing PC.

- Symptomatic patients who do not have PC are extremely valuable control subjects. These patients may present with abdominal pain, weight loss, and/or diarrhea; however, imaging of the pancreas is completely normal. When developing a biomarker for earlier detection of disease in symptomatic patients, this control group will be essential.

- Patients with newly diagnosed disease are those who have been diagnosed with PC.

- Patients undergoing treatment are those with PC undergoing treatment for their disease.

- Patients who fail therapy are those who have failed all therapies or who are not able to take additional therapies because of cumulative toxicities or disease progression.

\section{Patient Resources}

A key resource that is needed to enable biomarker studies is the availability of clinical samples from these different groups of subjects. Optimally, access would be available to lifelong history and longitudinal sampling of tissue and blood from all subjects, including samples prior to development of disease, during development of disease, at time of diagnosis, during treatment, and during disease progression following ineffective treatments. Finally, it would also be useful to have access to rapid autopsy samples at the time of death. The development of all these resources requires collaborative studies and significant funding.

Research in PC that required biospecimens was stagnant for decades because it was very difficult to ethically obtain annotated biospecimens because of rapid demise of patients and because the majority $(70 \%-80 \%)$ of PC patients were not surgically resected. Thus, investigators' access to material was piecemeal and not systematic. Much of the early work using biospecimens was based on opportunistic availability and small sample sizes, and hence, 
it was difficult to interpret or generalize findings. Several resources have been developed that partially address these issues.

\section{Registries}

Systematic information about PC at the national/state population level in the United States is available through the National Institutes of Health-funded SEER database, although biospecimens are not available. It may be possible through the state-based tumor registries and registries that contribute to SEER to obtain archival tissue from the $20 \%$ of patients who had their lesions resected, but this requires significant effort, time, and layers of approvals. Several registries of FPC are available. Listings of current FPC registries are provided by the Pancreatic Cancer Action Network at http://www.pancan.org/section-facing-pancreatic-cancer/learnabout-pan-cancer/risk-factors/risk-factors-family-registries/ and by Pancreatica at http://pancreatica.org/faq/pancreatic-cancer-registries/. The FPC registries generally identify families in which there are 2 or more FDRs with PC and/or family members who develop $\mathrm{PC}$ prior to age 50 years or in some cases have known syndromes that are associated with a higher incidence of PC. Registries (and often genetic studies) exist at the following centers or agencies in the United States: Creighton University, Columbia University, Dana Farber Cancer Center, Indiana University, Johns Hopkins, Mayo Clinic, Memorial Sloan Kettering Cancer Center, Mt Sinai Hospital-Icahn School of Medicine at Mt Sinai, New York Presbyterian Hospital, Weill Medical College of Cornell University, Northshore University Health Systems, Oregon Health and Science University, Thomas Jefferson University, University of Nebraska Medical Center, University of Oklahoma Health Sciences Center, University of Utah Huntsman Cancer Institute, and University of Washington. Internationally, there are registries at the Zane Cohen Center for Digestive Diseases of the Gastrointestinal Cancer Registry at Mt Sinai Hospital in Toronto, Ontario, Canada; at Phillips University of Marburg, Germany; the European Pancreatic Cancer Registry; and the Japanese Pancreatic Cancer Registry of the Japanese Pancreatic Society.

Among the university-based registries, there is some effort to collaborate. An example of this is PACGENE (Pancreatic Cancer Gene Consortium), ${ }^{138}$ organized by Mayo Clinic, and includes Johns Hopkins University, Dana-Farber Cancer Institute, Karmanos Cancer Institute Center at Wayne State, Creighton University, and MD Anderson Cancer Center.

Among the international registries, the PC registry of JPS, instituted in 1981, is the only nationwide registry of PC, sporadic as well as familial. These registries of patients with PC and their family members will provide new insights into the genetic, environmental, and lifestyle factors that may impose a risk of developing $\mathrm{PC}$.

In the United States, there are currently few nationwide efforts to establish registries for both sporadic and familial cases of PC. One such effort is the Pancreatic Cancer Collaborative Registry (PCCR; http://pccr.unmc.edu/). ${ }^{139}$ The PCCR, a multi-institutional Web-based system, was designed to collect data on PC patients and high-risk subjects through a standard and efficient online process. There are 7 cancer centers in the United States and 1 center in Italy participating in the PCCR, which currently contains data on more than 2700 subjects (PC patients and high-risk subjects).

\section{Clinic-Based Resources}

Mayo Clinic has established a unique Clinic-Based Registry and Biospecimen Resource. This resource unifies a PC patient registry with the tissue and biospecimen bank resources. In addition to the data and samples, a major and effective means of unifying the resource is through a database that has Web-based portals so that study coordinators and physicians can access the database throughout the Mayo Clinic campuses.

The Mayo Clinic Biospecimen Resource developed processes to address the shortcomings of the earlier era. Specific features of the resource that enable meaningful research include large volumes of patients; coordinated multidisciplinary clinical care and treatment via the Mayo Model of Care; searchable electronic schedules and access to electronic medical records; ultrarapid case finding and recruitment (approaching prospective patients prior to knowledge of diagnosis); inviting participation into research in person, rather than by mail; self-completed risk factor questionnaires; access to all treatment data; and follow-up to demise of patients.

Ultrarapid case finding and consenting to research are of paramount importance. In the setting of Mayo Clinic, $61 \%$ of patients approached agreed to participate in research (by 2014, 9380 potential subjects have been approached). When approached in person, $80 \%$ of patients agree to participate, compared with $27 \%$ when approached by mail. More importantly, $64.6 \%$ of 3574 patients with pancreatic adenocarcinoma were recruited within 2 days of diagnosis, and an additional $20 \%$ were recruited within 30 days of diagnosis. Because of the ultrarapid method of ascertainment, there is less likelihood of survival bias in research using samples from this resource, and indeed, samples can be assembled utilizing a variety of demographic, clinical, and tissue criteria. Patients are asked to provide $50-\mathrm{mL}$ research blood samples and to allow the registry to maintain contact for the following year. Blood samples are helpful for DNA-based studies, and in particular, it has been found that the major contribution can be in biomarker validation in serum or plasma. Potential biomarkers can be tested in a variety of different samples sets (distinguished by cancer vs noncancer diagnoses, history of DM, etc), and it can be documented that patient samples were drawn prior to the start of therapy. Approaching pancreatic clinic patients prior to confirmed diagnosis enables collection of disease control subjects (pancreatitis, neuroendocrine tumors, IPMN, cysts), which are collected under identical conditions as cases with PC.

For tissue-based studies, the resource has access to thousands of blocks of tissues, formalin fixed, paraffin embedded, and frozen, for which annotation has enabled high-quality analysis of samples. Tissue microarrays are available, which are organized by tumor characteristics (ie, adenocarcinoma vs IPMN). For a few samples, a recently initiated xenograft program has resulted in the construction of patient tumor cell lines of which more than a dozen have been genomically characterized by exome sequencing, expression array, and epigenetic analysis. Results of all assays performed are retained, enabling comparisons and cost savings in some cases by avoiding repeated analyses of the same markers on sets of samples.

Another example of intrainstitutional clinical collaboration to generate resources is the University of Michigan Multidisciplinary Pancreatic Cancer Clinic (MDP). This clinic sees about 700 patients yearly, of which approximately half have a diagnosis of pancreatic adenocarcinoma. Within the clinic, there is a fulltime research coordinator available to consent patients, collect blood, and arrange for tissue specimens to be obtained for various research programs at the university, including CTC analysis and detection, the primary human PC xenograft program, and biobanking. Within the past year, thanks to the research team being readily available in the clinic, the sample collection has been vastly increased, with more than 200 patients with pancreatic neoplasms already enrolled as of September 2014. Early Detection Research Network-based protocols are in place to collect blood samples over the course of treatment. Tissue is procured on all resection candidates and many biopsy samples. 
The MDP has also supported the development of a comprehensive clinical database of all patients seen in the clinic. While this was initially developed for quality assurance/quality improvement purposes, the use of this database has been expanded for research purposes. The MDP team has built a strong relationship with The University of Michigan Cancer Center informatics team that provides expertise and supports the technology needs by obtaining clinical data, integrating it into the database, and developing new bioinformatics tools.

The University of Michigan was included in the U01 grant resubmission of the PACGENE to be added as a new site. They have developed a program to provide genetic services and counseling to patients. The MDP clinic has fully integrated genetic services. A registry that collects DNA on patients with a personal and family history of cancer (about 4,000 subjects enrolled since 2002) is managed by The University of Michigan Cancer Genetics. This registry has also enrolled 40 families who meet criteria for familial pancreas cancer ( $\geq 2$ relatives with PC). By having the cancer genetics team integrated in clinic, recruitment numbers are expected to increase significantly, with $10 \%$ of PC cases being familial. In addition, the registry follows many families with germline mutations associated with PC risk, including CDKN2a, PRSS1, Peutz-Jeghers syndrome, Lynch syndrome (200+), and BRCA1 and BRCA2 (150+). Cancer Genetics maintains mechanisms for longitudinal followup with yearly questionnaires, as well as coordination of pancreatic screening.

There are several collaborative clinical resources at UCLA. The UCLA Center for Pancreatic Diseases (www.pancreas.ucla.edu) provides multidisciplinary care for a large volume of patients with pancreatic diseases. For the year 2011-2012, the total number of unique patients with all pancreatic diseases seen was 1716 , of whom 649 had PC, 517 had acute pancreatitis, 137 had CP, and 413 had pancreatic cystic disease. The UCLA Pancreas Tissue Bank has prospectively accrued more than 350 pairs of clinically annotated, snap-frozen pancreatic tumor and patient-matched normal tissues. In addition, the Pancreas Tissue Bank has generated more than 50 early-passage, patient-derived tumor xenografts, as well as associated primary cultures of PC and associated stroma. Available resources also include much larger numbers of archived, formalin-fixed diagnostic tissue blocks derived from FNAs, core biopsies, and surgically resected tissue samples of pancreatic neoplasms, both primary and metastatic, with available clinical annotations. Multiple tissue microarrays with robust clinicopathologic annotation are also available as high-throughput screening and validation tools. Southern California Research Center for Alcoholic Liver and Pancreatic Diseases and Cirrhosis, a National Institute on Alcohol Abuse and Alcoholism-funded center under the leadership of Drs Hidekazu Tsukamoto and Stephen J. Pandol, constitutes the first Los Angeles city-wide Center of Excellence for Studies of Alcohol and Liver Injury. The Ronald S. Hirshberg Translational Pancreatic Cancer Research Laboratory houses a current program project grant (Guido Eibl, PI, Targeting Diet Induced Promotion of Kras-Initiated Pancreatic Adenocarcinoma; http://dietandpancreaticcancer.com). In addition, the UCLA Center for Excellence in Pancreatic Diseases (http://www.pancreaticdiseasecenter.org/), under the leadership of Vay Liang W. (Bill) Go, constitutes a highly collaborative, multidisciplinary, and multi-institutional research program designed to make a significant impact on the investigation of complementary and alternative medicine in the prevention and therapy of pancreatic diseases. The center includes expertise from the UCLA campus, LA BioMed Research Institute at Harbor-UCLA Medical Center, and VA Greater Los Angeles Healthcare System-West Los Angeles. The Southern California Research Center for Alcoholic Liver and Pancreatic Disease is a National Institute on
Alcohol Abuse and Alcoholism-funded Alcohol Research Center, which is the first Los Angeles city-wide Center for Excellence devoted to Studies on Alcoholic Liver and Pancreatic Injury (http://www.usc.edu/schools/medicine/research/alcohol_center/). The center was created through the consolidation of existing collaborative and interactive programs among the established investigators at the University of Southern California (USC) and UCLA. The center has catalyzed cross-utilization of complementary resources by this center and other existing Centers of Excellence on the USC and UCLA campuses (USC Center for Liver Disease, USC Hepatitis C Cooperative Research Center, USC Norris Comprehensive Cancer Center, UCLA CURE Digestive Disease Research Center, and UCLA Center for Excellence in Pancreatic Diseases) to promote technical and academic synergism and to achieve integrated dissemination of educational activities.

The NIDDK Digestive Disease Core Center (CURE) (http://www.cure.med.ucla.edu/) is a digestive diseases research center composed of a cohesive group of physicians and basic scientists with strong independent, grant-supported research programs in the biology of the gut and pancreas. This center broadly investigates the physiology of the GI, liver, and pancreatic systems and the pathological conditions that impact these organs with the aim to translate scientific discovery into therapeutic interventions for patients.

\section{Rapid Autopsy Programs}

Two groups have been conducting rapid autopsies for PC for approximately the past 11 years (Nebraska and Johns Hopkins). Recently, a few other groups have initiated these programs. Rapid autopsy programs grew out of the need for tissue from PC patients, as it was evident that there is not a sufficient amount of surgically resected material to meet the needs of the research community. In addition to providing tissue, rapid autopsies that collect samples of primary tumor and all metastatic deposits allow the study of the entire molecular and pathological history of disease progression, as one can then sample individual parts of the primary tumor and compare these samples to distant metastases. ${ }^{15,140}$ This way, investigators can reconstruct molecular and cellular events that occurred as the disease progressed from initial phases to metastatic disease and death. In addition, rapid autopsies that capture other organs, whether involved with metastasis or not, enable studies of tumor host interaction, that is, studies to understand how the tumor interacted with other organ systems to cause the final demise of the patient. Although important and useful for obtaining valuable tissue, rapid autopsies become quite powerful when they are performed on patients for which there is also longitudinal tissue sampling and full annotation of the clinical history. For example, in the Nebraska series, there are surgically resected samples available for approximately $30 \%$ of the 87 autopsies done to date. Some of these patients have also been enrolled into clinical trials. Under these circumstances, the collection of longitudinal samples coupled with patient history enables a substantial number of additional studies, including biomarker studies, for either early detection or for markers of disease progression, response to therapy, or lack of response to therapy. ${ }^{141}$ These resources enable collaborative research in several ways. First, there is often a sufficient amount of material from the autopsy to provide samples for multiple investigators. Second, each cancer patient can be considered to be an experiment of nature. There are many variables in disease progression; however, by capturing different samples over time and by having available all tissues at death, one is able to utilize the patient's own tissues as controls for disease progression. Third, concerted efforts to compile experimental information from the study of autopsy and longitudinal samples will allow for efficient utilization of resources and could 
lead to synergistic findings. For example, compilation of DNA sequence, RNA sequencing information, and epigenetic analysis together with biological studies (signaling, pathology, other aspects of tumor behavior) for the same patient will enable investigators to more fully understand aspects of disease progression that are distinct or related to their particular field of study. This has a significant potential for synergistic effects in discovery.

Intraductal papillary mucinous neoplasm is reported to arise in the pancreas of pedigrees of FPC on follow-up before or at the development of PC. ${ }^{142}$ Even IPMN patients without a family history of PC may develop 1 or multiple concomitant PCs. Multiple IPMNs $(\geq 10)$ are associated with a higher prevalence of noninvasive or invasive concomitant PC. As these observations show increased predisposition of IPMN patients to PC, close surveillance of IPMN patients may lead to early detection of SPC. A JPS-endorsed group of 74 pancreas centers throughout Japan launched a prospective collaborative surveillance study in 2012 and registered more than 2100 patients with branch duct IPMN without "worrisome features" during a 2-year entry period until July 2014. These patients will undergo a fixed protocol of surveillance for 5 years, that is, blood tests, dynamic CT, magnetic resonance cholangiopancreatography, and EUS at the time of registry; blood tests plus CT every 6 months every year; and blood tests and magnetic resonance cholangiopancreatography plus EUS every 12 months. This study may allow us to determine precise incidence of PC concomitant with branch duct IPMN and provide us with some insight into the early diagnosis of SPC.

\section{Early Detection Research Network}

Early Detection Research Network of the NCI has established several key resources for researchers. First, the EDRN has established 2 reference sets: one that contains 100 sera and plasma from early-stage PCs along with 100 benign disease controls (other benign diseases of the pancreas) and a second, which is currently accruing samples, that contains sera and plasma from patients with resection-confirmed cystic lesions of the pancreas. These samples are all well annotated for clinical features and procured under a standard operating protocol. The collection of these samples required coordinated collaboration among more than 5 major pancreatic centers. A second significant contribution of the EDRN has been the publication of important principles and operating procedures for the systematic discovery and validation of biomarkers. ${ }^{143}$ The system consists of $(a)$ establishing a reference set of specimens collected under PRoBE design criteria, $(b)$ using the reference set to prevalidate candidate biomarkers before committing to full-scale validation, $(c)$ performing full-scale validation for those markers that pass prevalidation testing, and $(d)$ ensuring that the reference set is sufficiently large in numbers and volumes of sample that it can also be used to study future candidate biomarkers. This system provides rigorous and efficient evaluation of candidate biomarkers and biomarker panels. Reference sets should also be constructed to enable high-quality biomarker-discovery research. ${ }^{143}$

\section{Gaps in the Field}

1. Identification of high-risk populations and better biomarkers are needed for the early detection and diagnosis of $P C$. Diabetes, secondary to pancreatic diseases, is commonly referred to as type $3 \mathrm{C}$ diabetes. Approximately $75 \%$ of type $3 \mathrm{C}$ diabetes is due to $\mathrm{CP}$, which carries a high risk for pancreatic carcinoma. Determining the relationship between type $3 \mathrm{C}$ diabetes and the development of PC is an emerging area of study that is expected to influence the development of early detection methods. Questions that need to be addressed include the following: What is the link of other diseases in increasing the risk for $\mathrm{PC}$, especially type $3 \mathrm{C}$ diabetes? What are the links between $\mathrm{CP}$ and PC? Can biomarkers of risk or early stages of progression to PC be discovered and deployed?

Precursors of invasive ductal adenocarcinoma of the pancreas include PanINs, IPMNs, and mucinous cystic neoplasms. Pancreatic intraepithelial neoplasias are difficult to detect using current imaging modalities. However, these methods are increasingly detecting IPMNs in the pancreas. Clinically, the study of IPMNs that have the potential to progress to PC is important and has potential in early detection to identify asymptomatic patients.

2. A major gap in the field is the inability to utilize the unique resources of individual facilities across institutions/universities. Expertise and resources vary substantially between sites, and this limits opportunity to initiate the most robust science possible and negatively impacts advances in the field. In addition, there are barriers to collaboration across institutions that include competition for resources or publications, fear of intellectual theft or lack of credit for discoveries, pride and arrogance, or an attitude of isolation and independence that creates research silos.

3. A limited number of patients are enrolled in registry systems. For example, even the JPS PC registry (dealing with sporadic cancer as well as familial cancer) gathers only $10 \%$ to $15 \%$ of all patients with PC in Japan. Mere registration of SPC does not lead to early detection, whereas registration of FPC would give family members a chance for early detection of abnormal findings in their pancreas.

4. Another major gap in the field is the limited number of institutions that undertake longitudinal patient sampling from diagnosis until death and couple these to rapid autopsy programs. A better program would be the capacity to enroll patients who have not yet been diagnosed with PC into programs of research, in which they would donate clinical samples longitudinally, and if diagnosed with PC, during diagnosis and treatment, and then finally enroll in a rapid autopsy program at the end of life. Such a comprehensive program exists at the Mayo Clinic in a longitudinal program of prospective imaging of adult-onset diabetics. This program is unique and invaluable but limited in size by a lack of funding. Expansion of such a program would be a priority. In such a program, it is important to collate and annotate all related diagnostic information from these patients (ie, imaging, biomarkers, laboratory tests, etc). There should be a seamless consented enrollment of patients into these studies with patients agreeing from the beginning to participate in lifelong longitudinal studies of their disease. In addition, diagnostic tests could be incorporated into autopsy sampling. For example, one could obtain CTC from blood of patients at death, evaluate these for molecular and biological characteristics, and then evaluate the full spectrum of disease in the tissue samples to determine the extent to which the CTC accurately reflects the spectrum of disease in the patient. Another possible use of these samples for biomarker studies would be as a platform for characterizing the extent of expression of circulating biomarkers in serum or plasma; markers identified in discovery could be evaluated for extent and cell source of expression during disease progression. This analysis may also allow biomarker investigators to undertake biological studies of the biomarker or to integrate their studies collaboratively into ongoing biological studies by other investigators.

\section{Ideas and Opportunities}

- An inventory of resources available across the country that can be utilized collaboratively for PC research should be prepared. 
This PC resource dashboard could be accessed by participant investigators. Participants could opt into this consortium, agreeing to allow other participant investigators to utilize/share resources for projects and proposals.

- There should be mechanisms to increase collaboration among existing registries and centers that conduct specialized areas of research into different aspects of PC progression, including risk, development and detection of early disease, and progression of disease to metastasis.

- Centers should be established and/or expanded that are in a position to diagnose, treat, and enroll patients in longitudinal studies to obtain well-annotated clinical samples during the entire history of disease progression and treatment. These same centers should establish, participate in, or collaborate with centers that have ongoing surveillance or screening programs for PC (usually high-risk patients).

- The establishment and maintenance of biorepositories with specimens for use in $\mathrm{PC}$ research has been identified as a priority for future collaborative efforts. Specific recommendations include the collection of annotated specimens from patients with early stages of PC (stages IA and IB and stages IIA and IIB) and with benign pancreatic conditions (acute pancreatitis and $\mathrm{CP}$, biliary obstruction) and matching symptomatic control subjects.

- Development of more extensive and efficient registries of the general population to explore the possibility of early detection of SPC is needed. Although this risk has to be determined by genetic factors expected to be obtained by investigations of FPC, a more realistic collaborative study is urgently needed to increase the possibility of early detection of PC at present.

- There are very few biological samples from patients with primary PanIN-3 and IPMN with high-grade dysplasia (eg where no $\mathrm{PC}$ is present). Such patient samples will have a crucial role in the development of biomarkers and better methods of imaging. Resources need to be provided to focus on this valuable cohort of patients and collaborations need to be fostered to maximize use of these rare specimens.

- Biomarkers are needed to identify pancreatic precursor lesions with the potential to progress toward PC. This would include biomarkers to distinguish PanIN-3 from PanIN-1 and PanIN-2 and to distinguish IPMNs with high-grade dysplasia from those with low-grade dysplasia, which have a low risk of neoplastic progression.

- Collaborative and funding support for innovative imaging will be important, especially in the funding gap that exists between discovery of the method and the preclinical studies that are required to get an imaging agent through the Food and Drug Administration.

\section{Summary}

The development of effective methods for early detection requires committed collaboration of numerous scientific and clinical disciplines. It is anticipated that in the complicated field of early detection of SPC, the partnering of research institutions specializing in PC studies will afford the opportunity to share expertise and resources.

The establishment and maintenance of biorepositories with specimens for use in PC research have been identified as a priority for future collaborative efforts. Specific recommendations include the collection of specimens from patients with early stages of PC (stages IA and IB, and stages IIA and IIB), with PC precursor lesions (IPMNs, PanINs), and with benign pancreatic conditions (acute pancreatitis and CP, biliary obstruction). Determining the relationship between type $3 \mathrm{C}$ diabetes and the development of
PC is an emerging area of study that is expected to influence the development of early detection methods. In addition, the development of biomarkers to distinguish IPMNs with high risk of developing PC from those with low risk, the detection of early-stage PC and PanINs in patients at high risk, the use of panels of markers to identify profiles for precursor lesions associated with PC (IPMNs or PanIN), and imaging and biomarker development to stratify populations at risk for developing PC are projected areas for collaborative research.

\section{CONCLUSIONS}

Pancreatic ductal adenocarcinoma, commonly referred to as $\mathrm{PC}$, has a current 5 -year survival rate of only $6 \%$. This compares poorly to the National Institutes of Health report that the 5-year relative survival rate for breast cancer is $90 \%$, colorectal cancer is $67 \%$, and prostate cancer is nearing $100 \%$ (http://seer.cancer. gov/faststats/). Unequivocal evidence exists that the presymptomatic detection of earliest-stage PC can lead to significant improvement in the survival rate. Furthermore, even a reduction in the delay to diagnosis in symptomatic patients could improve survival.

Each invited scientific representative to the Kenner Family Research Fund's inaugural 2014 Early Detection of Sporadic Pancreatic Cancer Summit Conference was asked to contribute to this review of the major components impacting the development of early detection methods. The identified and analyzed gaps, challenges, and specific opportunities for innovation have provided a platform of knowledge that will be used to influence the collaborative future for breakthrough innovation. Substantive information has been presented within this article to inform scientific and clinical fields about the current state of specific areas of inquiry: Case for Early Detection: Definitions, Detection, Survival, and Challenges; Biomarkers for Early Detection; Imaging: and Collaborative Studies. An addendum containing information related to Screening in Familial Pancreatic Cancer is included with this article (Supplemental Digital Content 1, http://links.lww.com/MPA/A372).

Detection of PC prior to invasion is a primary goal of those experts participating in the summit. This will have a significant impact on survival when cases detected early will be amenable to treatment. The long, presymptomatic dwell time at both precancerous and early $\mathrm{T} 1$ cancer stages may actually provide a relatively wide window of opportunity for screening detection. While it remains cost-prohibitive and difficult with current technology to screen the general population for PC, models demonstrate that screening of individuals younger than 70 years and who have a lifetime risk of PC greater than or equal to $16 \%$ is cost-effective.

Targeted screening of asymptomatic, genetically predisposed individuals and screening of cohorts at high risk for SPC are approaches that may lead to improved mortality. Defining such high-risk groups that have a high likelihood of harboring such lesions, identifying biomarkers that could predict preinvasive cancers (carcinoma in situ) in high-risk cohorts, and developing imaging modalities that can detect such lesions are projected to have the greatest impact on early detection and survival.

While cohorts with high risk for PC in the setting of familial cancer are better defined, there are limited data on high-risk groups for sporadic (nonfamilial) PC. Subjects with CP and those with new-onset of diabetes after age 50 years are at higher-thanaverage risk for PC. However, in the absence of an enrichment strategy, there has been no progress in early detection in these cohorts.

Ideal screening markers would be universally present in advanced preinvasive cancer and curable-stage PC, while absent in those patients without neoplasia, such as pancreatitis. These biomarkers should be readily detectable in easily obtainable 
biosamples, ideally noninvasively captured and easily provided by the patient such as in body fluids (blood, saliva, stool) or other distant media (pancreatic juice). To encourage compliance and use, the assays should be rapid, inexpensive, widely distributable to maximize test access, and practical for assay. Most importantly, to be effective as a screening tool, they must be highly sensitive and have near-perfect specificity to accurately detect the critical target.

The ideal markers would reflect the molecular alterations that accompany the evolution from pancreatic precancer to preinvasive cancer and that result from exfoliated cells or secreted markers. These include overexpressed and underexpressed RNAs, mutations and other genetic derangements important in the biological progression, and various epigenetic changes, especially those involving aberrant methylation and proteins. Other markers may represent unique expression of glycosylated proteins and MUCs secreted by the cancers. Most recently, the detection and in-depth analysis of CPCs may offer diagnostic, predictive, and prognostic markers. Currently, there are many methodologies to capture these cells. While these technologies are innovative and compelling, much work still needs to be done to show that these methods are sensitive and specific compared with positive selection platforms, as well as establishing basic operating parameters that will allow for transfer to CLIA-certified labs.

Imaging techniques are also essential to confirm presence of early PC in subjects in whom biomarker studies predict their presence. Despite the lack of a universally accepted screening protocol, surveillance using EUS and/or MRI is well tolerated, and for individuals at high risk for PC, screening can find precancerous and early-stage disease. Emerging technologies may help to overcome the limitations of conventional EUS imaging. Although at varying stages of development and study, these technologies in clude contrast-enhanced EUS, elastography, and digital image analysis, as well as enhanced resolution for basic gray-scale imaging.

Molecular imaging has emerged as a potential modality to identify smaller lesions, translating into the potential to diagnose at a much earlier stage than is available. Specifically, molecular imaging has the benefit of being able to identify differences between tumor and normal or CP on a molecular level- not based on morphological differences. Being able to combine molecular imaging with conventional imaging, for example, molecular ultrasound, fluorescence endoscopy, or PET/MRI, could have important implications for patient outcomes.

The development of effective methods for early detection requires committed collaboration of numerous scientific and clinical disciplines. It is anticipated that in the complicated field of early detection of SPC, the partnering of research institutions specializing in PC studies will afford the opportunity to share expertise and resources. The establishment and maintenance of biorepositories with specimens for use in $\mathrm{PC}$ research has been identified as a priority for future collaborative efforts.

Understanding of the relationship between type 3C diabetes and the development of PC is an emerging area of study that is expected to influence the development of early detection methods. In addition, the development of biomarkers to distinguish IPMNs with high risk of developing PC from those with low risk, the detection of early-stage PC and PanINs in patients at high risk, the use of panels of markers to identify profiles for precursor lesions associated with PC, and imaging and biomarker development to stratify populations at risk for developing $\mathrm{PC}$ are projected areas for collaborative research.

The vast body of information presented in this article supports a new joint approach to meet identified gaps in the development of early detection methods for SPC. The complex nature of conducting collaborative scientific research is an influential driver in structuring the intentional model for the future of early detection.

This summative review of the scientific fields currently involved in early detection will serve as a critical component of the Early Detection of Sporadic Pancreatic Cancer Postsummit Conference white paper to be published in 2015. This report will include data analysis and outcomes from the seminal summit conference and will introduce the design of the strategic pathway for future interdisciplinary collaboration in the field. The white paper will present the strategic model for innovation that has been developed for these future collaborative efforts. Also included will be the component leadership, business, scientific, and funding priorities for the implementation of the new map. It is clear that significant progress cannot be made by silos of researchers but must be the result of strategically designed collaboration among individual investigators, institutions, and funders.

\section{ACKNOWLEDGMENTS}

The authors thank Dr Sonmoon Mohapatra for editing and reviewing the manuscript.

Modeling shown in Figure 1, Figure 2 and Table 1 was performed by Kenneth M. Boucher, PhD, Department of Oncological Sciences, University of Utah, Salt Lake City, Utah.

\section{REFERENCES}

References are available online at: http://links.lww.com/MPA/A374. 